\title{
A POSTERIORI ERROR ESTIMATES FOR VERTEX CENTERED FINITE VOLUME APPROXIMATIONS OF CONVECTION-DIFFUSION-REACTION EQUATIONS
}

\author{
MARIO OHLBERGER ${ }^{1}$
}

\begin{abstract}
This paper is devoted to the study of a posteriori error estimates for the scalar nonlinear convection-diffusion-reaction equation $c_{t}+\nabla \cdot(\mathbf{u} f(c))-\nabla \cdot(D \nabla c)+\lambda c=0$. The estimates for the error between the exact solution and an upwind finite volume approximation to the solution are derived in the $L^{1}$-norm, independent of the diffusion parameter $D$. The resulting a posteriori error estimate is used to define an grid adaptive solution algorithm for the finite volume scheme. Finally numerical experiments underline the applicability of the theoretical results.
\end{abstract}

Mathematics Subject Classification. 65M15, 35K65, 76M25.

Received: October 20, 2000. Revised: January 29, 2001.

\section{INTRODUCTION}

We consider the Cauchy problem of a degenerate convection-diffusion-reaction equation in $\mathbb{R}^{d}$, for $d=2,3$ :

$$
\begin{aligned}
& c_{t}+\nabla \cdot(\mathbf{u} f(c))-\nabla \cdot(D(c) \nabla c)+\lambda c=0 \quad \text { in } \mathbb{R}^{d} \times(0, T), \\
& c(\cdot, 0)=c_{0} \quad \text { in } \mathbb{R}^{d} .
\end{aligned}
$$

Here $\mathbf{u}: \mathbb{R}^{d} \times \mathbb{R}^{+} \rightarrow \mathbb{R}^{d}$ denotes a given velocity, $f: \mathbb{R} \rightarrow \mathbb{R}$ a nonlinear function, $D(c) \geq 0$ denotes the diffusion parameter and $\lambda$ the reaction coefficient.

In this paper we continue our work on a posteriori error estimates for finite volume approximations of nonlinear conservation laws and convection diffusion equations, which was started in [33] and [41]. In [41] we analyzed an explicit cell centered finite volume approximation to an unstationary convection diffusion equation and proved an a posteriori error estimator of order $\left(\varepsilon^{2}+h+\Delta t\right)^{1 / 4}$, where $\varepsilon$ denoted the small diffusion parameter $(e . g . \quad \varepsilon \leq h)$. It must be mentioned that in the case of a dominating, strictly positive diffusion parameter, where we can assume regularity of the exact solution of the problem, an error bound of order $h+\Delta t$ can be shown with energy methods ( $c f$. [3]). In those estimates the constants do heavily depend on the diffusion parameter, such that they break down for $D=\varepsilon \rightarrow 0$. Thus in the study here, we are always interested in the case, where the diffusion coefficient is either very small, or degenerate. The following analysis can be seen as an improvement of the result, obtained in [41], as we get a uniform bound on the error, which is completely

\footnotetext{
Keywords and phrases. A posteriori error estimates, convection diffusion reaction equation, finite volume schemes, adaptive methods, unstructured grids.

1 Institut für Angewandte Mathematik, Universität Freiburg, Hermann-Herderstr. 10, 79104 Freiburg, Germany. e-mail: mario@mathematik. uni-freiburg.de
} 
independent of the size of the diffusion coefficient. The improvement of the a posteriori error estimate in this paper could be obtained by exploiting the latest results concerning uniqueness and continuous dependence for degenerate parabolic equations $[8,15,20,31]$. The crucial point and difference to the technique used in [41] is that we exploit the positive second order term on the right hand side in the definition of the entropy solution (cf. Def. 2.4). Hence we need that gradients of the approximate solution are well defined. Therefore, we choose vertex centered finite volume schemes for the analysis in this paper instead of cell centered schemes. The choice of an implicit scheme has no special relevance for the proof of the a posteriori error estimate. Indeed, the estimates in this chapter do directly apply also in the explicit case if an appropriate CFL-condition is assumed. Finally, we chose the implicit scheme for the demonstration of the proof because the freedom of the choice of the time step $\Delta t$ is an additional challenge for the development of an adaptive solution strategy.

Equations of the type (1) are of special interest as they model physical motions in which the convective fluxes dominate the diffusive ones. Such problems occur for example in fluid dynamics with high Reynolds numbers [32], in density driven ground-water flow or in immiscible two-phase flow problems in porous media [40]. Another rather important application is the transport of contaminants in subsurface flow models [25].

Many authors studied convection-diffusion equations and proposed different numeric solution techniques, such as non-conforming finite elements $[16,17,30,47]$, streamline diffusion methods [29,30], combined finite volume - finite element methods [3,28] or finite volume schemes [26,34]. See also [44] for an overview on finite element methods and exponentially fitted schemes.

In contrast to the situation for finite volume schemes, the a posteriori theory for finite element discretizations of parabolic problems is very well developed. Here we refer to $[4,18,19,46]$. We also mention the more heuristic feed-back approach [6]. All these articles are concerned with elliptic or parabolic equations and the $a$ posteriori error estimates depend exponentially on the inverse of the diffusion parameter. In [27] an robust a posteriori error estimates for Lagrange-Galerkin schemes in the $\mathrm{H}^{-1}$-norm is derived. An energy norm a posteriori error estimate for linear stationary convection-diffusion equations, discretised via finite element methods, is given in [47]. But the error estimate in this work is only optimal if the local mesh-Peclet number is sufficiently small which means that the mesh size must be at least of the same order as the diffusion parameter. Especially, the results break down for the limiting case where $D$ tends to zero. In [38] an a posteriori error estimate for a characteristic Galerkin approximation of linear unstationary convection-diffusion equations is deduced. Further results on a posteriori error estimates for finite element approximations were obtained in [39] for degenerate parabolic equations including the classical Stefan problem. In [2] linear stationary convection dominated anisotropic diffusion problems are considered and error estimates in the energy norm are derived.

The techniques used in this paper go back [35] and [36]. In the context of nonlinear hyperbolic conservation laws these techniques where used to prove a priori estimates $[9,10,12,14,21,45,48]$ and a posteriori estimates $[13$, $33,41]$. In the context of degenerate parabolic equations these techniques where recently successfully used in $[8,31]$. Based on the uniqueness result of this last named works, convergence of an implicit finite volume scheme for nonlinear degenerate parabolic equations on bounded domains was recently proved in [22]. Let us remark that a priori error estimates are still an open problem in this situation. We also point out that the problem (1), which is discussed in this paper may be seen as a scalar case of weakly coupled systems, as they are studied in the hyperbolic framework in [42] and [43]. Thus we plan to extend the results of this work to weakly coupled systems in a forthcoming paper.

In the sequel the paper will be organized as follows. In Section 2 some properties of the exact solution of (1) are stated, whereas in Section 3 the approximate solution $c_{h}$ is defined ( $c f$. Def. 3.5) and some assumptions for the following analysis are given. The discrete scheme is analyzed in Section 4, where an entropy inequality for the approximate solution is shown and some stability results are given. Finally in Section 5 the a posteriori error estimate of the form $\left\|c_{h}-c\right\|_{L^{1}\left(\mathbb{R}^{d} \times(0, T)\right)} \leq \eta\left(c_{h}\right)$ is proved for the implicit cell centered finite volume scheme (cf. Th. 5.4). In Section 6 we deduce an adaptation strategy from the a posteriori error estimates and justify the theoretical results by some numerical experiments. 


\section{Properties And Regularity of the exact SOlution}

In order to define proper notations for solutions of problem (1), we state the following assumptions on the data.

Assumption 2.1. For the data of problem (1) we assume the following conditions

$$
\begin{aligned}
c_{0} & \in L^{\infty}\left(\mathbb{R}^{d}\right) \cap W^{1,1}\left(\mathbb{R}^{d}\right), \quad f \in C^{2}(\mathbb{R}), f, f^{\prime}, f^{\prime \prime} \in L^{\infty}(\mathbb{R}) \\
\mathbf{u} & \in C^{1}\left(\mathbb{R}^{d} \times \mathbb{R}^{+}\right)^{d} \cap L^{\infty}\left(\mathbb{R}^{d} \times \mathbb{R}^{+}\right)^{d} \text { with } \nabla \cdot \mathbf{u}=0, \\
\lambda & \in C^{1}\left(\mathbb{R}^{d} \times \mathbb{R}^{+}\right),\|\lambda\|_{L^{\infty}\left(\mathbb{R}^{d} \times \mathbb{R}^{+}\right)} \leq M, \quad D \in C^{1}(\mathbb{R}), \text { with } D(s) \geq 0, \quad \forall s \in \mathbb{R} .
\end{aligned}
$$

Following the guidelines of Carrillo [8] and Karlsen, Risebro [31] we can now define weak solutions and entropy solutions of problem (1).

Definition 2.2 (Weak solution). Let the Assumptions 2.1 be fulfilled. Then a weak solution of problem (1) is a measurable function $c$ satisfying

$$
\begin{array}{r}
c \in L^{1}\left(\mathbb{R}^{d} \times \mathbb{R}^{+}\right), \quad \partial_{t} c \in L^{2}\left(0, T ; H^{-1}\left(\mathbb{R}^{d}\right)\right), \\
d(c):=\int_{0}^{c} D(s) \mathrm{d} s \in L^{2}\left(0, T ; H^{1}\left(\mathbb{R}^{d}\right)\right), \quad f(c) \in L^{2}\left(\mathbb{R}^{d} \times \mathbb{R}^{+}\right), \\
c_{t}+\nabla \cdot(\mathbf{u} f(c))-\nabla \cdot(D(c) \nabla c)+\lambda c=0 \text { in } \mathcal{D}^{\prime}\left(\mathbb{R}^{d} \times(0, T)\right), \\
c(\cdot, 0)=c_{0}, \text { a.e. in } \mathbb{R}^{d} .
\end{array}
$$

Remark 2.3. The existence of a weak solution can be shown ( $c f$. [8]), but in general, the definition of a weak solution does not guarantee the uniqueness. Thus, we will define an entropy solution to problem (1) which will then give us also uniqueness. In the sequel we will always refer to the entropy solution, when we speak of the solution of problem (1).

Definition 2.4 (Entropy solution). Let $U$ be a smooth even entropy function and $F_{U}$ a corresponding entropy flux $\left(e . g . \partial_{v} F_{U}(v, \kappa)=f^{\prime}(v) \partial_{v} U(v-\kappa)\right.$ for $\kappa \in \mathbb{R}$ fixed). An entropy solution of (1) is a weak solution $c$ satisfying

$$
\begin{array}{r}
\left.\int_{\mathbb{R}^{d} \times R^{+}} U(c-\kappa) \partial_{t} \varphi+\left(F_{U}(c, \kappa) \mathbf{u}-D(c) U^{\prime}(c-\kappa) \nabla c\right) \cdot \nabla \varphi-\lambda c U^{\prime}(c-\kappa)\right) \varphi+\int_{\mathbb{R}^{d}} U\left(c_{0}-\kappa\right) \varphi(\cdot, 0) \\
\geq \int_{\left\{(x, t) \in \mathbb{R}^{d} \times \mathbb{R}^{+} \mid D(c(x, t))>0\right\}} D(c) U^{\prime \prime}(c-\kappa)|\nabla c|^{2} \varphi
\end{array}
$$

for all nonnegative $\varphi \in \mathcal{D}\left([0, T) \times \mathbb{R}^{d}\right)$ and all $\kappa \in \mathbb{R}$.

Remark 2.5. We remark that the definition of an entropy solution is independent of the choice of $U$, as all smooth even entropies can be approximated by linear combinations of $U(\cdot-\kappa), \kappa \in \mathbb{R}$.

Theorem 2.6 (Existence and uniqueness of entropy solutions). Let the Assumptions 2.1 be fulfilled. Then there exists a unique entropy solution $c$ as defined in 2.4 .

Proof. The existence and uniqueness of entropy solutions in the degenerate case was first shown by Carrillo [8] on bounded domains and then extended to the Cauchy-problem by Karlsen, Risebro [31], by assuming even weaker regularity on the data.

Although all the techniques which are used in this paper are designed to treat the general nonlinear situation, where the diffusion parameter $D$ depends on the solution $c$, we will only consider the case $D \equiv$ const in the sequel for simplicity. 
Assumption 2.7. Let the Assumptions 2.1 be fulfilled and let the diffusion parameter $D$ be independent of the solution $c$, such that $D \in \mathbb{R}$, with $D>0$.

Remark 2.8. We point out that all the estimates which are shown in the sequel hold independently of the size of $D$. Therefore, the main a posteriori result (Th. 5.4) holds also in the case $D \equiv 0$, where all terms involving $D$ vanish (at least formally). In this way the error bounds are robust in $D$.

In order to state the next theorem, let as introduce the space of bounded variation. Therefore we first define for $g \in L^{1}\left(\mathbb{R}^{d}\right)$ the BV-semi-norm as

$$
|g|_{B V}:=\sup _{\varphi \in C_{c}^{1}\left(\mathbb{R}^{d}\right)^{d},\|\varphi\|_{\infty} \leq 1} \int_{\mathbb{R}^{d}} g \nabla \cdot \varphi \mathrm{d} x .
$$

The space of functions with bounded variation is then defined as $B V\left(\mathbb{R}^{d}\right):=\left\{\left.g \in L^{1}\left(\mathbb{R}^{d}\right)|| g\right|_{B V}<\infty\right\}$.

Theorem 2.9 (Estimates independent of the diffusion parameter $D$ ). Let $c$ be the entropy solution of problem (1) and let the Assumptions 2.7 be fulfilled. Then $c(\cdot, t) \in W^{1,1}\left(\mathbb{R}^{d}\right)$ for all $t>0$ and the following estimates hold for all $t, t_{1}, t_{2} \geq 0$, where the constants $K_{1}, K_{2}$ and $K_{3}$ do not depend on $D$.

$$
\begin{aligned}
\|c\|_{L^{\infty}\left(\mathbb{R}^{d} \times(0, T)\right)} & \leq\left\|c_{0}\right\|_{L^{\infty}\left(\mathbb{R}^{d}\right)} \mathrm{e}^{M T} \\
\|c(\cdot, t)\|_{W^{1,1}\left(\mathbb{R}^{d}\right)} & \leq K_{1}\left\|c_{0}\right\|_{W^{1,1}\left(\mathbb{R}^{d}\right)}+K_{2}, \\
\int_{\mathbb{R}^{d}}\left|c\left(\cdot, t_{1}\right)-c\left(\cdot, t_{2}\right)\right| & \leq K_{3}\left\|c_{0}\right\|_{W^{1,1}\left(\mathbb{R}^{d}\right)}\left|t_{2}-t_{1}\right|,
\end{aligned}
$$

where $M:=\|\lambda\|_{L^{\infty}\left(\mathbb{R}^{d} \times(0, T)\right)}$. In the limiting case, where $D=0$, these inequalities hold for the $B V$-norm instead of $W^{1,1}$.

Proof. The proof of this estimates within an even more general framework is given in Kruzkov [35]. See also [37] for a survey of the methods used and [42] for the estimates in the case of weakly coupled systems of parabolic and hyperbolic type.

\section{Notations, ASSumptions And Definition of the SCHEME}

In this section we will fix the notations and assumptions and define the implicit vertex centered upwind finite volume scheme for solving (1). In order to reduce the complexity of the notations we will restrict in the sequel to the two dimensional case, i.e. we will assume $d=2$ for our further considerations. Nevertheless all the techniques used in this paper will also hold in the three dimensional case. The only difference to two dimensions is the construction of a dual partition of a tetrahedral grid in three dimensions which would force us to introduce an even more complex notation ( $c f$. [1] for the construction of dual cells in 3D and the definition of the corresponding finite volume scheme).

Let $J:=\left\{t_{0}, \ldots, t_{N}\right\}$ be a partition of $[0, T]$ and $\Delta t^{n}:=t^{n+1}-t^{n}$ the step size of $J$. Furthermore, let $\mathcal{T}:=\left\{T_{j} \mid j \in K\right\}$ be an admissible triangulation of $\mathbb{R}^{2}$, where $K$ denotes an index set of the triangles, i.e. $\mathcal{T}$ covers the domain $\mathbb{R}^{2}$ and each set $T_{j} \cap T_{l}, j, l \in K, j \neq l$ is either empty or a common $(2-k)$-simplex, $k \in\{1,2\}$. The joint edge of two neighbouring vertices $p_{j}$ and $p_{l}$ will be denoted by $\Gamma_{j l}$ and $I$ denotes the set of vertex indices of the triangulation ( $c f$. Fig. 1 ). The oriented normal vector to $\Gamma_{j l}$ will be denoted by $\mathbf{m}_{j l}$. By $\mathcal{E}$ we denote the oriented set of faces, i.e.

$$
\mathcal{E}:=\left\{(j, l) \in I \times I \mid \Gamma_{j l} \text { is the edge connecting the points } p_{j}, p_{l} \text { and } j>l\right\} .
$$

Define for each vertex $p_{j}, j \in I$ of the triangulation $\mathcal{T}$ the corresponding dual cell $\Omega_{j}$ by connecting the centers of gravity of the surrounding triangles with the center of gravities of the edges $\Gamma_{j l}$. The resulting curve is the contour line of the dual volume $\Omega_{j}$ (cf. Fig. 1 for an illustration in 2D). The mesh of dual cells $\Omega:=\left\{\Omega_{j} \mid j \in I\right\}$ 

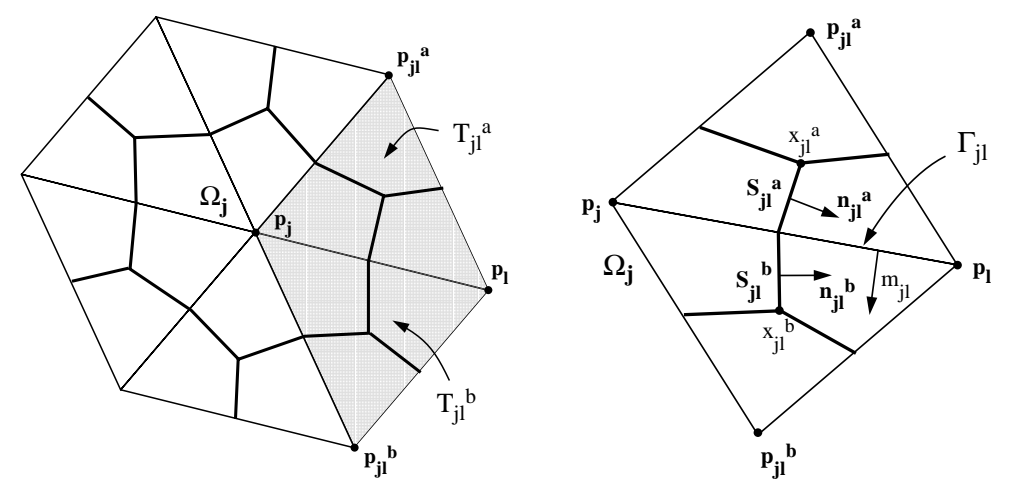

FiguRE 1. Notations for the triangulation $\mathcal{T}$ and the dual partition $\Omega$

is a partition of our domain $\mathbb{R}^{2}$ and serves as the finite volumes in our numerical method. Let $N(j) \subset I$ indicate the indices of neighbouring cells of the dual cell $\Omega_{j}$.

Let us indicate values above the edge $\Gamma_{j l}$ by an upper index $a$ and values below $\Gamma_{j l}$ by an upper index $b$ and let $\mathcal{P} M:=\{a, b\}$. More precise, above is defined by a counter clockwise orientation of the vertices $p_{j}, p_{l}, p_{j l}^{a}$ and below by a clockwise orientation of the vertices $p_{j}, p_{l}, p_{j l}^{b}$ (cf. Fig. 1). Thus, the joint edges of $\Omega_{j}$ and $\Omega_{l}$ will be denoted by $S_{j l}^{a}, S_{j l}^{b}$. The unit outer normal vector to $S_{j l}^{a}, S_{j l}^{b}$ with respect to $\Omega_{j}$ will be denoted by $\mathbf{n}_{j l}^{a}, \mathbf{n}_{j l}^{b}$. In the same manner we denote by $T_{j l}^{a}, T_{j l}^{b}$ the adjacent triangles of the edges $\Gamma_{j l}$.

Furthermore, let us denote $h_{j}:=\operatorname{diam}\left(\Omega_{j}\right), h_{j l}:=\operatorname{diam}\left(\Omega_{j} \cup \Omega_{l}\right)$ and $h_{\min }:=\min _{j \in I} h_{j}, h:=\max _{j \in I} h_{j}$. Finally, let $\mathcal{E}^{*}$ denote the oriented set of edges defined as

$$
\mathcal{E}^{*}:=\left\{(j, l, *) \in I \times I \times \mathcal{P} M \mid S_{j l}^{*} \text { is a common edge of the dual cells } \Omega_{j} \text { and } \Omega_{l} \text { lying in } T_{j l}^{*} \text { and } j>l\right\} \text {. }
$$

Assumption 3.1. We assume that the triangulation $\mathcal{T}$ is weakly acute (no triangle with an angle greater then $\pi / 2)$. Then there exists an $\alpha>0$ such that we have for all $h_{j}, j \in I$

$$
\alpha h_{j}^{d} \leq\left|\Omega_{j}\right|, \quad \alpha\left|\partial \Omega_{j}\right| \leq h_{j}^{d-1} .
$$

\subsection{The finite volume scheme}

In the sequel we will define an implicit vertex centered finite volume approximation of problem (1), where we restrict to the case given in Assumption 2.7. In order to do so we first define numerical fluxes for the convective and diffusive part of the problem.

Convective fluxes: For any $j, l \in I, a \in \mathcal{P} M$ and $t_{n} \in \mathbb{R}^{+}$, let $g_{j l}^{a, n} \in C^{1}\left(\mathbb{R}^{2}, \mathbb{R}\right)$ be a numerical convective flux, satisfying the following conditions for all $w, v, w^{\prime}, v^{\prime} \in[A, B]$, where $A, B \in \mathbb{R}$ are chosen such that $A \leq c \leq B$ :

$$
\partial_{w} g_{j l}^{a, n}(w, v) \geq 0, \quad \partial_{v} g_{j l}^{a, n}(w, v) \leq 0 .
$$

Furthermore, there exists a constant $L_{g}>0$ independent of $j, l, n$ and $h$, such that for all $w, v, w^{\prime}, v^{\prime}$

$$
\begin{aligned}
g_{j l}^{a, n}(w, v) & =-g_{l j}^{b, n}(v, w) \\
\left|g_{j l}^{a, n}(w, v)-g_{j l}^{a, n}\left(w^{\prime}, v^{\prime}\right)\right| & \leq L_{g}\left|S_{j l}^{a}\right|\left(\left|w-w^{\prime}\right|+\left|v-v^{\prime}\right|\right), \\
g_{j l}^{a, n}(w, w) & =\frac{1}{\Delta t^{n}} \int_{t^{n}}^{t^{n+1}} \int_{S_{j l}^{a}} \mathbf{u}(x, t) \cdot n_{j l}^{a} \mathrm{~d} x \mathrm{~d} t f(w),
\end{aligned}
$$


where $n_{j l}^{a}$ denotes the outer unit normal to $S_{j l}^{a}$ with respect to $\Omega_{j}$. The corresponding conditions should hold for $g_{j l}^{b, n}: \mathbb{R}^{2} \rightarrow \mathbb{R}$ as well. Note that $\sum_{\substack{l \in N(j), * \in \mathcal{P} M}} g_{j l}^{*, n}(s, s)=0$, since $\operatorname{div} \mathbf{u}=0$.

Remark 3.2. This class of convective fluxes are called monotone upwind fluxes in conservation form. Examples are the Lax-Friedrichs or Enquist-Osher fluxes ( $c f .[32]$ for an overview).

Diffusive fluxes: Let $V_{h} \subset H^{1}\left(\mathbb{R}^{2}\right)$ be the space of piecewise linear functions on $\mathcal{T}$ which are globally continuous. For any $j, l \in I, * \in \mathcal{P} M$ and $t_{n} \in \mathbb{R}^{+}$let the diffusive numerical fluxes $d_{j l}^{*, n}: V_{h} \rightarrow \mathbb{R}$ be defined as

$$
d_{j l}^{*, n}\left(w_{h}\right)=\int_{S_{j l}^{*}} D \nabla w_{h}^{n} \cdot n_{j l}^{*} \mathrm{~d} x \mathrm{~d} t
$$

for any $w_{h}^{n} \in V_{h}$. Note that $d_{j l}^{*, n}\left(w_{h}^{n}\right)$ does depend on $\left.w_{h}^{n}\right|_{T_{j l}^{*}}$ only .

Remark 3.3. Let the Assumption 3.1 be fulfilled. Then it can be shown, that $d_{j l}^{*, n}$ can be written as

$$
d_{j l}^{*, n}\left(w_{h}\right)=-D_{j l}^{*, n}\left(w_{j}-w_{l}\right), \quad D_{j l}^{*, n}:=\left|S_{j l}^{*}\right| D \frac{1}{2}\left(\nabla N_{l}-\nabla N_{j}\right) \cdot \mathbf{n}_{j l}^{*},
$$

where $D_{j l}^{*, n} \in \mathbb{R}$ is positive and symmetric in $j, l$. Here $N_{j}, N_{l}$ denote the base functions of the finite element space $V_{h}$ with respect to the points $p_{j}, p_{l}$ in the triangle $T_{j l}^{*}$. (A proof can be found in [1].)

In the following lemma we state the equivalence between the finite element and finite volume formulation of the diffusive part.

Lemma 3.4. Let $\mathcal{T}$ be an admissible triangulation. Then for each $j \in I, T \in \mathcal{T}$ and $v_{h} \in V_{h}$ the following identity holds

$$
\int_{T} \nabla v_{h} \cdot \nabla N_{j} \mathrm{~d} x=-\int_{\partial \Omega_{j} \cap T} \nabla v_{h} \cdot n \mathrm{~d} \gamma
$$

where $N_{j}$ denotes the base function of $V_{h}$ corresponding to $p_{j}$ and $n$ denotes the outer unit normal on $\partial \Omega_{j}$.

Proof. The proof is based on the geometric relations between the triangles and the dual cells and can be found, for instance, in [1], Lemma 13.

Now the upwind finite volume scheme for computing the approximate solutions to (1) is defined by

Definition 3.5 (Finite volume scheme (implicit)). Let $V_{h} \subset H^{1}\left(\mathbb{R}^{2}\right)$ be the space of piecewise linear functions on $\mathcal{T}$ which are globally continuous. Then for each $n \in\{0, \ldots, N\}$ the approximate solution $c_{h}^{n} \in V_{h}$ is given by the nodal basis coefficients $c_{j}^{n}, j \in I$, defined as:

$$
c_{j}^{0}:=\frac{1}{\left|\Omega_{j}\right|} \int_{\Omega_{j}} c_{0}, \quad c_{j}^{n+1}+\frac{\Delta t^{n}}{\left|\Omega_{j}\right|} \sum_{l \in N(j)} \sum_{* \in \mathcal{P} M}\left\{g_{j l}^{*, n+1}\left(c_{j}^{n+1}, c_{l}^{n+1}\right)-d_{j l}^{*, n+1}\left(c_{h}^{n+1}\right)\right\}+\Delta t^{n} \lambda_{j}^{n+1} c_{j}^{n+1}=c_{j}^{n} .
$$

for all $n \in\{0, \ldots, N\}$ and $j, l \in I$. Here $N(j)$ denotes the indices of the neighbouring cells of $\Omega_{j}$ and $\lambda_{j}^{n+1}$ are the mean values of $\lambda$ over $\Omega_{j} \times\left(t^{n}, t^{n+1}\right)$.

With this definition we further define the space-time function $c_{h}$ by:

$$
c_{h}(\cdot, 0):=c_{h}^{0}, \quad c_{h}(\cdot, t):=c_{h}^{n+1} \quad \text { for all } t \in\left(t^{n}, t^{n+1}\right] .
$$


Assumption 3.6 (Stability condition (implicit)). For the time step $\Delta t^{n}$ of the implicit scheme 3.5 we assume the following stability condition for $M:=\|\min \{\lambda, 0\}\|_{L^{\infty}\left(\mathbb{R}^{d} \times \mathbb{R}^{+}\right)}$and a given $\beta \in(0,1)$.

$$
\min _{\substack{j \in I, t_{n} \in J}}\left(1+\Delta t^{n} \lambda_{j}^{n+1}\right) \geq 1-\Delta t^{n} M \geq \beta
$$

\section{Properties of the Discrete SOlutions}

In order to establish an error analysis for the convection diffusion equation (1), we first study the behaviour of the discrete solution. Therefore, we first show that the discrete solution as defined in Definition 3.5 is $L^{\infty}$ stable. Similar results for the problem without reaction (e.g. $\lambda=0$ ) where obtained in [23] and [24], whereas in [10] stability in the case of a conservation laws with source term was shown for a semi-implicit cell centered finite volume scheme. To obtain this result we follow the guidelines of [21]. For the "local" existence and the stability estimate we use a result of Fuhrmann and Langmach, which uses that the Jacobian of the nonlinear mapping is an M-Matrix (cf. [24]).

Lemma 4.1 (Existence and uniqueness and $L^{\infty}$-stability). Let the stability condition 3.6 be fulfilled. Then there exists a unique discrete solution $c_{h}$ of 3.5 with the following properties.

1) $\left\|c_{h}\right\|_{L^{\infty}\left(\mathbb{R}^{2} \times\left(0, t_{n}\right)\right)} \leq\left\|c_{0}\right\|_{L^{\infty}\left(\mathbb{R}^{2}\right)} \prod_{n=0}^{N} \frac{1}{1-\Delta t^{n} M}$, for all $t_{n} \in J$.

2) If the initial values $c_{0}$ are nonnegative, then for all times the discrete solution $c_{h}$ is nonnegative.

Here $M:=\|\min \{\lambda, 0\}\|_{L^{\infty}\left(\mathbb{R}^{d} \times \mathbb{R}^{+}\right)}$.

Proof.

\section{Step 1: Uniqueness}

The proof follows by induction over the time steps. From Definition 3.5 it is clear, that the initial values $c_{h}^{0}$ are unique. Now let us assume that a solution $c_{h}^{n}$ in $L^{\infty}\left(\mathbb{R}^{2}\right)$ is given. Furthermore, let $c_{h}^{n+1}, s_{h}^{n+1}$ be two solutions of the discrete scheme (12) for given $c_{h}^{n}$ which fulfill the bound $\left\|c_{h}^{n+1}\right\|_{\infty},\left\|s_{h}^{n+1}\right\|_{\infty} \leq \frac{1}{1-\Delta t^{n} M}\left\|c_{h}^{n}\right\|_{L^{\infty}\left(\mathbb{R}^{2}\right)}$. If we subtract the equations defining $c_{h}^{n+1}, s_{h}^{n+1}$, we get:

$$
\begin{aligned}
\frac{\left|\Omega_{j}\right|}{\Delta t^{n}}\left(c_{j}^{n+1}-s_{j}^{n+1}\right)\left(1+\Delta t^{n} \lambda_{j}^{n+1}\right)= & -\sum_{\substack{l \in N(j), * \in \mathcal{P} M}} g_{j l}^{*, n+1}\left(c_{j}^{n+1}, c_{l}^{n+1}\right)+g_{j l}^{*, n+1}\left(s_{j}^{n+1}, s_{l}^{n+1}\right) \\
& -\sum_{\substack{l \in N(j), * \in \mathcal{P} M}} D_{j l}^{*, n}\left(c_{j}^{n+1}-c_{l}^{n+1}-s_{j}^{n+1}+s_{l}^{n+1}\right) .
\end{aligned}
$$

As $1+\Delta t^{n} \lambda_{j}^{n+1} \geq 1-\Delta t^{n} M>0$ (Assum. 3.6) we can divide by this term and get

$$
\begin{aligned}
\frac{\left|\Omega_{j}\right|}{\Delta t^{n}}\left(c_{j}^{n+1}-s_{j}^{n+1}\right)= & \frac{1}{2\left(1+\Delta t^{n} \lambda_{j}^{n+1}\right)} \\
& \times \sum_{\substack{l \in N(j), * \in \mathcal{P} M}}\left(2 g_{j l}^{*, n+1}\left(s_{j}^{n+1}, s_{l}^{n+1}\right)-g_{j l}^{*, n+1}\left(c_{j}^{n+1}, s_{l}^{n+1}\right)-g_{j l}^{*, n+1}\left(s_{j}^{n+1}, c_{l}^{n+1}\right)\right. \\
& +g_{j l}^{*, n+1}\left(s_{j}^{n+1}, c_{l}^{n+1}\right)+g_{j l}^{*, n+1}\left(c_{j}^{n+1}, s_{l}^{n+1}\right)-2 g_{j l}^{*, n+1}\left(c_{j}^{n+1}, c_{l}^{n+1}\right) \\
& \left.+2 D_{j l}^{*, n}\left(s_{j}^{n+1}-c_{j}^{n+1}-\left(s_{l}^{n+1}-c_{l}^{n+1}\right)\right)\right) .
\end{aligned}
$$


Let us define $\varphi(x):=\mathrm{e}^{-\kappa|x|}$, for some $\kappa>0$ which will be defined in the sequel. Furthermore, let $\varphi_{j}:=$ $\frac{1}{\left|\Omega_{j}\right|} \int_{\Omega_{j}} \varphi(x) \mathrm{d} x$. Multiplying the equation by $\varphi_{j}$ and rearranging the summands we get

$$
\begin{aligned}
\frac{\left|\Omega_{j}\right| \varphi_{j}}{\Delta t^{n}}\left(c_{j}^{n+1}-s_{j}^{n+1}\right)= & \frac{\varphi_{j}}{2\left(1+\Delta t^{n} \lambda_{j}^{n+1}\right)} \\
& \times \sum_{\substack{l \in N(j), * \in \mathcal{P M M}}}\left(\left(\bar{\partial}_{1} g_{j l}^{*, n+1}\left(s_{j}^{n+1}, c_{j}^{n+1} ; s_{l}^{n+1}\right)+\bar{\partial}_{1} g_{j l}^{*, n+1}\left(s_{j}^{n+1}, c_{j}^{n+1} ; c_{l}^{n+1}\right)\right)\left(s_{j}^{n+1}-c_{j}^{n+1}\right)\right. \\
& \left.-\left(\bar{\partial}_{2} g_{j l}^{*, n+1}\left(s_{j}^{n+1} ; s_{l}^{n+1}, c_{l}^{n+1}\right)+\bar{\partial}_{2} g_{j l}^{* n+1}\left(c_{j}^{n+1} ; s_{l}^{n+1}, c_{l}^{n+1}\right)\right)\left(s_{l}^{n+1}-c_{l}^{n+1}\right)\right)
\end{aligned}
$$

where $\bar{\partial}_{1} g_{j l}^{*, n+1}(u, v ; w)$ and $\bar{\partial}_{2} g_{j l}^{*, n+1}(w ; u, v)$ are defined as 0 for $u=v$ and for $u \neq v$ as

$$
\bar{\partial}_{1} g_{j l}^{*, n+1}(u, v ; w):=D_{j l}^{*, n}+\frac{g_{j l}^{* n+1}(u, w)-g_{j l}^{*, n+1}(v, w)}{u-v}, \quad \bar{\partial}_{2} g_{j l}^{*, n+1}(w ; u, v):=D_{j l}^{*, n}-\frac{g_{j l}^{* n+1}(w, u)-g_{j l}^{* n+1}(w, v)}{u-v} .
$$

Let us remark that using the properties of the numerical fluxes $g_{j l}^{*, n+1}$ and the Definition of $D_{j l}^{*, n}$ we have $\bar{\partial}_{1} g_{l j}^{*, n+1}(u, v ; w)=\bar{\partial}_{2} g_{j l}^{*, n+1}(w ; u, v) \geq 0$. Summing up over $j \in I$ we see with the same arguments as in [21, Prop. 3.1] that the series of the resulting equation are convergent and can be reordered. Therefore, we get by rearrangement of the summation

$$
\begin{aligned}
\sum_{j \in I} \frac{\left|\Omega_{j}\right| \varphi_{j}}{\Delta t^{n}}\left|c_{j}^{n+1}-s_{j}^{n+1}\right| \leq & \frac{1}{2 \gamma} \sum_{j \in I}\left|c_{j}^{n+1}-s_{j}^{n+1}\right| \\
& \times \sum_{\substack{l \in N(j), * \in \mathcal{P} M}}\left(\left(\bar{\partial}_{1} g_{j l}^{*, n+1}\left(s_{j}^{n+1}, c_{j}^{n+1} ; s_{l}^{n+1}\right)+\bar{\partial}_{1} g_{j l}^{*, n+1}\left(s_{j}^{n+1}, c_{j}^{n+1} ; c_{l}^{n+1}\right)\right)\left|\varphi_{j}-\varphi_{l}\right|\right) .
\end{aligned}
$$

Defining $a_{j}$ and $b_{j}$ as

$$
a_{j}:=\frac{\left|\Omega_{j}\right| \varphi_{j}}{\Delta t^{n}}, \quad b_{j}:=\frac{1}{2\left(1-\Delta t^{n} M\right)} \sum_{\substack{l \in N(j), * \in \mathcal{P M}}}\left(2\left(D_{j l}^{*, n}+L_{g}\left|S_{j l}^{*}\right|\right)\left|\varphi_{j}-\varphi_{l}\right|\right)
$$

this yields with the Lipschitz continuity of $g_{j l}^{*, n+1}$

$$
\sum_{j \in I} a_{j}\left|c_{j}^{n+1}-s_{j}^{n+1}\right| \leq \sum_{j \in I} b_{j}\left|c_{j}^{n+1}-s_{j}^{n+1}\right| .
$$

Now we recall that $\varphi(x):=\mathrm{e}^{-\kappa|x|}$ and we choose $\kappa$ small enough in order to have $a_{j}>b_{j}$ for all $j \in I$. This is possible because for any given constant $K$ we can choose $\kappa$ such that the inequality

$$
\inf _{y \in B_{h}(x)} \varphi(y) \geq K \sup _{y \in B_{2 h}(x)}|\nabla \varphi(y)|
$$

holds for all $x \in \mathbb{R}^{2}$. Therefore, we have shown that $c_{j}^{n+1}=s_{j}^{n+1}$ which finishes the uniqueness proof.

\section{Step 2: Existence and stability}

In this step we will show by induction over the time steps that there exists a discrete solution $c_{h}$ of 3.5 with the stability properties 1) and 2) of the Lemma. First of all it is clear from the definition that there exists a $c_{h}^{0}$ with the proposed properties. Now let $c_{h}^{n}$ be given with a bound $\left\|c_{h}^{n}\right\|_{L^{\infty}\left(\mathbb{R}^{2}\right)} \leq A_{n}$, for a given constant $A_{n}>0$. We will show that there exists a solution $c_{h}^{n+1}$, given by the scheme (12) with the stability bound $\left\|c_{h}^{n+1}\right\|_{\infty} \leq \frac{1}{1-\Delta t^{n} M} A_{n}$. Furthermore, we show that if $c_{h}^{n}$ is nonnegative, then $c_{h}^{n+1}$ is nonnegative as well. 
In order to prove this result we first consider a finite dimensional subproblem. Therefore, let us define $c_{h}^{(r)}$ for some $r \in \mathbb{N}$ large enough. For all $j$, such that $\Omega_{j} \not \subset B_{r}(0)$ we define $c_{j}^{(r)}:=c_{j}^{n}$ and for all $j$, such that $\Omega_{j} \subset B_{r}(0)$ let $c_{j}^{(r)}$ be defined as the solution of the following equation

$$
c_{j}^{(r)}+\frac{\Delta t^{n}}{\left|\Omega_{j}\right|} \sum_{l \in N(j)} \sum_{* \in \mathcal{P} M}\left\{g_{j l}^{*, n+1}\left(c_{j}^{(r)}, c_{l}^{(r)}\right)-d_{j l}^{*, n+1}\left(c_{h}^{(r)}\right)\right\}+\Delta t^{n} \lambda_{j}^{n+1} c_{j}^{(r)}=c_{j}^{n} .
$$

We will now prove that there exists $\left\{c_{j}^{(r)} \mid \Omega_{j} \subset B_{r}(0)\right\}$ and that $c_{h}^{(r)}$ fulfills the bound $\left\|c_{h}^{(r)}\right\|_{\infty} \leq \frac{1}{1-\Delta t^{n} M} A_{n}$. In order to do so, we write equation (14) in the following form

$$
\left|\Omega_{j}\right| c_{j}^{(r)}+\Delta t^{n} \sum_{l \in N(j)} \sum_{* \in \mathcal{P} M}\left\{g_{j l}^{*, n+1}\left(c_{j}^{(r)}, c_{l}^{(r)}\right)-d_{j l}^{*, n+1}\left(c_{h}^{(r)}\right)\right\}=\frac{\left|\Omega_{j}\right|}{1+\Delta t^{n} \lambda_{j}^{n+1}} c_{j}^{n} .
$$

As this finite dimensional nonlinear system of equations fulfills all assumptions of Theorem 5.10 in [24] we can use exactly the proof of Theorem 5.10 in [24] which gives us the existence and uniqueness of a solution $\left\{c_{j}^{(r)} \mid \Omega_{j} \subset B_{r}(0)\right\}$, satisfying (14) and the estimate $\left\|c_{h}^{(r)}\right\|_{\infty} \leq \frac{1}{1-\Delta t^{n} M}\left\|c_{h}^{n}\right\|_{\infty} \leq \frac{1}{1-\Delta t^{n} M} A_{n}$. Furthermore, we get if $c_{h}^{n}$ is nonnegative that $c_{h}^{(r)}$ is nonnegative as well. (Remark that the proof of this result in [24] is done by showing that the Jacobian of the nonlinear mapping, is a M-matrix.)

As we have proven the result for the finite dimensional problem, we are now going to pass to the limit $r \rightarrow \infty$. For $r \in \mathbb{N}$, let $\left\{c_{j}^{(r)}\right\}_{j \in I}$ be the solution of the finite dimensional problem above. As we have a uniform $L^{\infty}$ bound with respect to $r$ for this sequence of solutions, we can extract, by a diagonal procedure, a subsequence $r_{l}, l \in \mathbb{N}$ with $r_{l} \rightarrow \infty$ such that $\left\{c_{j}^{\left(r_{l}\right)}\right\}_{j \in I}$ is convergent and the limit fulfills the same stability bounds as the solutions $\left\{c_{j}^{\left(r_{l}\right)}\right\}_{j \in I}$. Now let $c_{j}^{n+1}$ be defined as $c_{j}^{n+1}:=\lim _{l \rightarrow \infty} c_{j}^{\left(r_{l}\right)}$. Passing to the limit in equation (14) shows, that the limit $c_{h}^{n+1}$ is solution of (12). Now, using the uniqueness of a solution, we get that $c_{h}^{n+1}$ is the limit for the whole sequence $\left\{c_{h}^{(r)}\right\}_{r \in \mathbb{N}}$. This completes the proof of Lemma 4.1.

In the next step we establish entropy inequalities for the approximate solution ( $c f$. Def. 3.5). Therefore, we first introduce the entropies and correlated fluxes we want to consider here. Following the guidelines of Cockburn and Gremaud [14] we introduce the entropies $U=U_{\delta}$ which are approximations of the Kruzkov-entropies and the correlated entropy fluxes $F_{U}$.

Definition 4.2 (Entropy $U$ and entropy-flux $\left.F_{U}\right)$. Let $\bar{U} \in C^{2}\left(\mathbb{R}, \mathbb{R}^{+}\right)$be an even function, such that

$$
\bar{U}(0)=0, \quad \bar{U}^{\prime}(v) \begin{cases}=1 & \text { if } v \geq 1, \\ \in[-1,1] & \text { if }-1<v<1, \quad \bar{U}^{\prime \prime} \geq 0 . \\ =-1 & \text { if } v \leq-1,\end{cases}
$$

For any $\delta>0, v \in \mathbb{R}$ let us define $U: \mathbb{R} \rightarrow \mathbb{R}^{+}$by $U(v):=\delta \bar{U}\left(\frac{v}{\delta}\right)$. Furthermore, define $F_{U}: \mathbb{R} \rightarrow \mathbb{R}$ for any $v, \kappa \in \mathbb{R}$ by $F_{U}(v, \kappa):=\int_{\kappa}^{v} f^{\prime}(w) U^{\prime}(w-\kappa) \mathrm{d} w$.

Lemma 4.3. Let $U, F_{U}$ be defined as above. Then the following inequalities hold for any $v, \kappa \in \mathbb{R}$

$$
\begin{aligned}
|v|-k_{0} \delta \leq U(v) & \leq|v| \\
\left|\partial_{v}\left(F_{U}(v, \kappa)-F_{U}(\kappa, v)\right)\right| & \leq \frac{k_{1}}{2} \delta\left\|f^{\prime \prime}\right\|_{L^{\infty}(\mathbb{R})}
\end{aligned}
$$

where $k_{0}:=\sup _{|w| \leq 1}|| w|-\bar{U}(w)|$ and $k_{1}:=\sup _{|w| \leq 1} \bar{U}^{\prime \prime}(w)$.

Proof. Cf. Cockburn and Gremaud [14]. 
Definition 4.4 (The discrete entropy flux). Let $U$ be defined as in 4.2. For any $j, l \in I, a \in \mathcal{P} M$ and $t_{n} \in \mathbb{R}^{+}$, let $G_{j l}^{a, n} \in C^{1}\left(\mathbb{R}^{2}, \mathbb{R}\right)$ be a discrete entropy flux correlated to the entropy $U$. Let us assume that $G_{j l}^{a, n}$ satisfies for all $w, v, w^{\prime}, v^{\prime} \in[A, B]$, where $A, B \in \mathbb{R}$ are chosen such that $A \leq c \leq B$ :

$$
\partial_{w} G_{j l}^{a, n}(w, v) \geq 0, \quad \partial_{v} G_{j l}^{a, n}(w, v) \leq 0 .
$$

Furthermore, there exists a constant $L_{G}>0$ independent of $j, l, n$ and $h$, such that for all $w, v, w^{\prime}, v^{\prime}$

$$
\begin{aligned}
G_{j l}^{a, n}(w, v) & =-G_{l j}^{b, n}(v, w), \\
\left|G_{j l}^{a, n}(w, v)-G_{j l}^{a, n}\left(w^{\prime}, v^{\prime}\right)\right| & \leq L_{G}\left|S_{j l}^{a}\right|\left(\left|w-w^{\prime}\right|+\left|v-v^{\prime}\right|\right), \\
G_{j l}^{a, n}(w, w) & =\frac{1}{\Delta t^{n}} \int_{t^{n}}^{t^{n+1}} \int_{S_{j l}^{a}} \mathbf{u}(x, t) \cdot n_{j l}^{a} \mathrm{~d} x \mathrm{~d} t F_{U}(w, \kappa)
\end{aligned}
$$

and additionally the following compatibility condition should hold

$$
\partial_{v} G_{j l}^{a, n}(w, v)=\partial_{v} g_{j l}^{a, n}(w, v) U^{\prime}(v-\kappa)
$$

where $n_{j l}^{a}$ denotes the outer unit normal to $S_{j l}^{a}$ with respect to $\Omega_{j}$. The corresponding conditions should hold for $G_{j l}^{b, n}: \mathbb{R}^{2} \rightarrow \mathbb{R}$ as well. Note that $\sum_{\substack{l \in N(j) \\ * \in \mathcal{P} M}} G_{j l}^{*, n}(s, s)=0$, since $\operatorname{div} \mathbf{u}=0$.

Remark 4.5. For the construction of the discrete entropy fluxes correlated to the entropy $U$ and the entropy flux $F_{U}$ cf. Lemma 3 and Lemma 4 in [9].

Definition 4.6 (The discrete form $\left.E_{j}^{n}\right)$. For any $v_{h} \in L^{\infty}\left(0, T ; V_{h}\right), \kappa \in \mathbb{R}$ let us define

$$
\begin{aligned}
E_{j}^{n}\left(c_{h}, \kappa\right):= & U\left(c_{j}^{n+1}-\kappa\right)-U\left(c_{j}^{n}-\kappa\right)+\frac{\Delta t^{n}}{\left|\Omega_{j}\right|} \sum_{\substack{l \in N(j), * \in \mathcal{P} M}} G_{j l}^{*, n+1}\left(c_{j}^{n+1}, c_{l}^{n+1}\right) \\
& +\frac{\Delta t^{n}}{\left|\Omega_{j}\right|} \sum_{l \in N(j)} D \int_{T_{j l}^{a}} \nabla c_{h}^{n+1} \cdot \nabla N_{j} U^{\prime}\left(c_{j}^{n+1}-\kappa\right)+\Delta t^{n} \lambda_{j}^{n+1} c_{j}^{n+1} U^{\prime}\left(c_{j}^{n+1}-\kappa\right), \\
F_{j}^{n}\left(c_{h}, \kappa\right):= & \int_{c_{j}^{n}}^{c_{j}^{n+1}} \int_{s}^{c_{j}^{n+1}} U^{\prime \prime}(w-\kappa) \mathrm{d} w \mathrm{~d} s+\frac{\Delta t^{n}}{\left|\Omega_{j}\right|} \sum_{\substack{l \in N(j) \\
* \in \mathcal{P M}}} \int_{c_{j}^{n+1}}^{c_{l}^{n+1}} \partial_{w} g_{j l}^{*, n+1}\left(c_{j}^{n+1}, w\right) \int_{w}^{c_{j}^{n+1}} U^{\prime \prime}(s-\kappa) \mathrm{d} s \mathrm{~d} w,
\end{aligned}
$$

where $N_{j}$ denotes the base function of $V_{h}$ corresponding to $p_{j}, U$ is defined as in 4.2 and $G_{j l}^{*, n+1}$ are the discrete entropy fluxes, defined in 4.4 .

Lemma 4.7 (Discrete entropy equality). Let $c_{h}$ be the approximate solution of (1), defined in 3.5 and let the Assumptions 2.7, 3.1 be fulfilled. Then with the Definition 4.6 we have for all $j \in I$ :

$$
E_{j}^{n}\left(c_{h}, \kappa\right)=-F_{j}^{n}\left(c_{h}, \kappa\right) \leq 0 .
$$

Proof. If we multiply (12) by $U^{\prime}\left(c_{j}^{n+1}-\kappa\right)$ we get:

$$
\begin{aligned}
\mathrm{RHS}:= & c_{j}^{n+1} U^{\prime}\left(c_{j}^{n+1}-\kappa\right)-c_{j}^{n} U^{\prime}\left(c_{j}^{n+1}-\kappa\right)+\frac{\Delta t^{n}}{\left|\Omega_{j}\right|} \sum_{\substack{l \in N(j), * \in \mathcal{P M}}} g_{j l}^{*, n+1}\left(c_{j}^{n+1}, c_{l}^{n+1}\right) U^{\prime}\left(c_{j}^{n+1}-\kappa\right) \\
& -\frac{\Delta t^{n}}{\left|\Omega_{j}\right|} \sum_{\substack{l \in N(j), * \in \mathcal{P M}}} d_{j l}^{*, n+1}\left(c_{h}^{n+1}\right) U^{\prime}\left(c_{j}^{n+1}-\kappa\right)+\Delta t^{n} \lambda_{j}^{n+1} c_{j}^{n+1} U^{\prime}\left(c_{j}^{n+1}-\kappa\right)=0 .
\end{aligned}
$$


Applying Definition (9) and Lemma 3.4 to the third term of RHS, we get

$$
\begin{aligned}
\mathrm{RHS}= & c_{j}^{n+1} U^{\prime}\left(c_{j}^{n+1}-\kappa\right)-c_{j}^{n} U^{\prime}\left(c_{j}^{n+1}-\kappa\right)+\frac{\Delta t^{n}}{\left|\Omega_{j}\right|} \sum_{\substack{l \in N(j), * \in \mathcal{P M}}} g_{j l}^{*, n+1}\left(c_{j}^{n+1}, c_{l}^{n+1}\right) U^{\prime}\left(c_{j}^{n+1}-\kappa\right) \\
& +\frac{\Delta t^{n}}{\left|\Omega_{j}\right|} \sum_{l \in N(j)} D \int_{T_{j l}^{a}} \nabla c_{h}^{n+1} \cdot \nabla N_{j} U^{\prime}\left(c_{j}^{n+1}-\kappa\right)+\Delta t^{n} \lambda_{j}^{n+1} c_{j}^{n+1} U^{\prime}\left(c_{j}^{n+1}-\kappa\right) .
\end{aligned}
$$

Now it can be easily seen, that $F_{j}^{n}\left(c_{h}, \kappa\right)$ is given by RHS $-E_{j}^{n}\left(c_{h}, \kappa\right)$, where we use for the first term of RHS the identity $U^{\prime}(b-\kappa)(b-a)=U(b-\kappa)-U(a-\kappa)+\int_{a}^{b} \int_{s}^{b} U^{\prime \prime}(w-\kappa) \mathrm{d} w \mathrm{~d} s$. Furthermore, we used the properties (5)-(8) and (17)-(21) of the fluxes $g_{j l}^{*, n+1}$ and $G_{j l}^{*, n+1}$ respectively, in order to get for the second term of RHS

$$
\begin{aligned}
\sum_{\substack{l \in N(j), * \in \mathcal{P M}}} g_{j l}^{*, n+1}\left(c_{j}^{n+1}, c_{l}^{n+1}\right) U^{\prime}\left(c_{j}^{n+1}-\kappa\right)=\sum_{\substack{l \in N(j), * \in \mathcal{P} M}}\left(g_{j l}^{*, n+1}\left(c_{j}^{n+1}, c_{l}^{n+1}\right)-g_{j l}^{*, n+1}\left(c_{j}^{n+1}, c_{j}^{n+1}\right)\right) U^{\prime}\left(c_{j}^{n+1}-\kappa\right) \\
=\sum_{\substack{l \in N(j), * \in \mathcal{P} M}} \int_{c_{j}^{n+1}}^{c_{l}^{n+1}} \partial_{w} g_{j l}^{*, n+1}\left(c_{j}^{n+1}, w\right) U^{\prime}\left(c_{j}^{n+1}-\kappa\right) \mathrm{d} w \\
=\sum_{\substack{l \in N(j), * \in \mathcal{P} M}} \int_{c_{j}^{n+1}}^{c_{l}^{n+1}} \partial_{w} g_{j l}^{*, n+1}\left(c_{j}^{n+1}, w\right) U^{\prime}(w-\kappa) \mathrm{d} w \\
\quad+\sum_{\substack{l \in N(j) \\
* \in \mathcal{P} M}} \int_{c_{j}^{n+1}}^{c_{l}^{n+1}} \partial_{w} g_{j l}^{*, n+1}\left(c_{j}^{n+1}, w\right)\left(U^{\prime}\left(c_{j}^{n+1}-\kappa\right)-U^{\prime}(w-\kappa)\right) \mathrm{d} w \\
=\sum_{\substack{l \in N(j, j \\
* \in \mathcal{P} M}} G_{j l}^{*, n+1}\left(c_{j}^{n+1}, c_{l}^{n+1}\right)+\sum_{\substack{l \in N(j) \\
* \in \mathcal{P} M}} \int_{c_{j}^{n+1}}^{c_{l}^{n+1}} \partial_{w} g_{j l}^{*, n+1}\left(c_{j}^{n+1}, w\right) \int_{w}^{c_{j}^{n+1}} U^{\prime \prime}(s-\kappa) \mathrm{d} s \mathrm{~d} w .
\end{aligned}
$$

As $U^{\prime \prime}$ and $-\partial_{w} g_{j l}^{*, n+1}(v, w)$ are positive we get $F_{j}^{n}\left(c_{h}, \kappa\right) \geq 0$ as well, which completes the proof.

Definition 4.8 (The form E). For any $v_{h} \in L^{\infty}\left(0, T ; V_{h}\right), \kappa \in \mathbb{R}$ let us define

$$
\begin{aligned}
E\left(v_{h}, \kappa\right):= & \int_{\mathbb{R}^{2} \times R^{+}} U\left(v_{h}(x, t)-\kappa\right) \partial_{t} \Phi(x, t) \mathrm{d} x \mathrm{~d} t+\int_{\mathbb{R}^{2}} U\left(c_{0}(x)-\kappa\right) \Phi(x, 0) \mathrm{d} x \\
& +\int_{\mathbb{R}^{2} \times R^{+}} F_{U}\left(v_{h}(x, t), \kappa\right) \mathbf{u}(x, t) \cdot \nabla \Phi(x, t) \mathrm{d} x \mathrm{~d} t \\
& -\int_{\mathbb{R}^{2} \times R^{+}} D\left(U^{\prime}\left(v_{h}(x, t)-\kappa\right) \nabla v_{h}(x, t) \cdot \nabla \Phi(x, t)+U^{\prime \prime}\left(v_{h}(x, t)-\kappa\right)\left|\nabla v_{h}(x, t)\right|^{2} \Phi(x, t)\right) \mathrm{d} x \mathrm{~d} t \\
& -\int_{\mathbb{R}^{2} \times R^{+}} \lambda(x, t) v_{h}(x, t) U^{\prime}\left(v_{h}(x, t)-\kappa\right) \Phi(x, t) \mathrm{d} x \mathrm{~d} t,
\end{aligned}
$$

where $\Phi \in C_{0}^{\infty}\left(\mathbb{R}^{2} \times \mathbb{R}^{+} ; \mathbb{R}^{+}\right)$is a smooth nonnegative test function.

Lemma 4.9 (Continuous entropy inequality for the discrete solution). Let $c_{h}$ be the approximate solution of (1), defined in 3.5 and let the Assumptions 2.7, 3.1 and 3.6 be fulfilled. Then with the Definition 4.8 we have

$$
E\left(c_{h}, \kappa\right) \geq-R_{h}\left(c_{h}\right),
$$


where $R_{h}\left(c_{h}\right)$ is given by

$$
\begin{aligned}
& R_{h}\left(c_{h}\right):=\sum_{t^{n} \in J} \sum_{j \in I}\left|c_{j}^{n+1}-c_{j}^{n}\right| \int_{t^{n}}^{t^{n+1}} \int_{\Omega_{j}} \int_{0}^{1}\left|\partial_{t} \Phi\left(x, t^{n}+\theta\left(t-t^{n}\right)\right)\right| \mathrm{d} \theta \mathrm{d} x \mathrm{~d} t+\int_{\mathbb{R}^{2}}\left|c_{0}(x)-c_{h}(x, 0)\right| \Phi(x, 0) \mathrm{d} x \\
& +\sum_{t^{n} \in J} \sum_{j \in I} h_{j} \int_{\Omega_{j}}\left|\nabla c_{h}\left(x, t^{n+1}\right)\right| \int_{t^{n}}^{t^{n+1}}\left|\partial_{t} \Phi(x, t)\right| \mathrm{d} x \mathrm{~d} t \\
& +\sum_{t^{n} \in J(j, l, *) \in \mathcal{E}^{*}} C_{j l}^{*, n+1}\left(c_{j}^{n+1}, c_{l}^{n+1}\right)\left|c_{j}^{n+1}-c_{l}^{n+1}\right|\left(h_{j}+\Delta t^{n}\right) \Delta t^{n}\left\langle\mu_{j l}^{*, n},\left|\partial_{t} \Phi\right|+|\nabla \Phi|\right\rangle \\
& +\sum_{t^{n} \in J(j, l, *) \in \mathcal{E}^{*}} C_{l j}^{*, n+1}\left(c_{l}^{n+1}, c_{j}^{n+1}\right)\left|c_{j}^{n+1}-c_{l}^{n+1}\right|\left(h_{l}+\Delta t^{n}\right) \Delta t^{n}\left\langle\mu_{l j}^{*, n},\left|\partial_{t} \Phi\right|+|\nabla \Phi|\right\rangle \\
& +\sum_{t^{n} \in J(j, l, *) \in \mathcal{E}^{*}} L_{f}\left|c_{j}^{n+1}-c_{l}^{n+1}\right|\left(\Delta t^{n}+h_{j l}\right)\left\langle\nu_{j l}^{*, n},|\nabla \Phi|+\left|\partial_{t} \Phi\right|\right\rangle \\
& +\sum_{t^{n} \in J} \sum_{j \in I}\|\mathbf{u}\|_{L^{\infty}\left(\Omega_{j} \times\left(t^{n}, t^{n+1}\right]\right)} L_{f} h_{j} \int_{\Omega_{j}}\left|\nabla c_{h}\left(x, t^{n+1}\right)\right| \int_{t^{n}}^{t^{n+1}}|\nabla \Phi(x, t)| \mathrm{d} x \mathrm{~d} t \\
& +\sum_{t^{n} \in J} \sum_{(j, l) \in \mathcal{E}} D\left[\nabla c_{h}^{n+1} \cdot \mathbf{m}_{j l}\right]_{\Gamma_{j l}}\left(\frac{2}{\delta}|| \bar{U}^{\prime \prime} \|_{L^{\infty}(\mathbb{R})}\left|c_{j}^{n+1}-c_{l}^{n+1}\right| \int_{t^{n}}^{t^{n+1}} \int_{\Gamma_{j l}} \Phi(\gamma, t) \mathrm{d} \gamma \mathrm{d} t\right. \\
& \left.+\Delta t^{n}\left|\Gamma_{j l}\right|\left(h_{j l}^{n}+\Delta t^{n}\right)\left(\left\langle\mu_{j l}^{n},\left|\partial_{t} \Phi\right|+|\nabla \Phi|\right\rangle+\left\langle\mu_{l j}^{n},\left|\partial_{t} \Phi\right|+|\nabla \Phi|\right\rangle\right)\right) \\
& +\sum_{t^{n} \in J} \sum_{j \in I}\left|c_{j}^{n+1}\right| \int_{t^{n}}^{t^{n+1}} \int_{\Omega_{j}} \Phi(x, t)\left|\lambda_{j}^{n+1}-\lambda(x, t)\right| \mathrm{d} x \mathrm{~d} t \\
& +\sum_{t^{n} \in J} \sum_{j \in I}\|\lambda\|_{L^{\infty}\left(\Omega_{j} \times\left(t^{n}, t^{n+1}\right]\right)}\left(1+\left|c_{j}^{n+1}\right| \frac{1}{\delta}\left\|\bar{U}^{\prime \prime}\right\|_{L^{\infty}(\mathbb{R})}\right) h_{j} \int_{\Omega_{j}}\left|\nabla c_{h}\left(x, t^{n+1}\right)\right| \int_{t^{n}}^{t^{n+1}} \Phi(x, t) \mathrm{d} t \mathrm{~d} x .
\end{aligned}
$$

Here $L_{f}$ denotes the Lipschitz constant of $f$ and we used the notations

$$
C_{j l}^{*, n+1}(v, w):=\frac{g_{j l}^{*, n+1}(v, w)-g_{j l}^{*, n+1}(v, v)}{(v-w)}, \quad\left[\nabla c_{h}^{n+1} \cdot \mathbf{m}_{j l}\right]_{\Gamma_{j l}}:=\left|\left(\left.\nabla c_{h}^{n+1}\right|_{T_{j l}^{a}}-\left.\nabla c_{h}^{n+1}\right|_{T_{j l}^{b}}\right) \cdot \mathbf{m}_{j l}\right|
$$

where $C_{j l}^{*, n+1}(v, w)$ is positive, because of the monotony of the numerical flux. Furthermore the measures $\mu_{j l}^{n}, \mu_{j l}^{*, n}$ are defined as

$$
\begin{aligned}
\left\langle\mu_{j l}^{n}, g\right\rangle:= & \frac{1}{\left(\Delta t^{n}\right)^{2}\left|\Omega_{j}\right|\left|\Gamma_{j l}\right|} \int_{t^{n}}^{t^{n+1}} \int_{\Omega_{j}} \int_{t^{n}}^{t^{n+1}} \int_{\Gamma_{j l}} \int_{0}^{1} g(x+\theta(\gamma-x), \tau+\theta(t-\tau)) \mathrm{d} \theta \mathrm{d} \gamma \mathrm{d} s \mathrm{~d} x \mathrm{~d} t, \\
\left\langle\nu_{j l}^{*, n}, g\right\rangle:= & \frac{1}{\left(\Delta t^{n}\right)^{2}\left|S_{j l}^{*}\right|^{2}} \int_{t^{n}}^{t^{n+1}} \int_{S_{j l}^{*}}\left(\int_{t^{n}}^{t^{n+1}} \int_{S_{j l}^{*}}\left|(\mathbf{u}(\gamma, t)-\mathbf{u}(\xi, \tau)) \cdot \mathbf{n}_{j l}^{*}\right| \mathrm{d} \xi \mathrm{d} \tau\right. \\
& \left.\times \int_{t^{n}}^{t^{n+1}} \int_{S_{j l}^{*}} \int_{0}^{1} g(\xi+\theta(\gamma-\xi), \tau+\theta(t-\tau)) \mathrm{d} \theta \mathrm{d} \xi \mathrm{d} \tau\right) \mathrm{d} \gamma \mathrm{d} t, \\
\left\langle\mu_{j l}^{*, n}, g\right\rangle:= & \frac{1}{\left(\Delta t^{n}\right)^{2}\left|\Omega_{j}\right|\left|S_{j l}^{*}\right|} \int_{t^{n}}^{t^{n+1}} \int_{\Omega_{j}} \int_{t^{n}}^{t^{n+1}} \int_{S_{j l}^{*}} \int_{0}^{1} g(x+\theta(\gamma-x), \tau+\theta(t-\tau)) \mathrm{d} \theta \mathrm{d} \gamma \mathrm{d} s \mathrm{~d} x \mathrm{~d} t .
\end{aligned}
$$


Proof. From Lemma 4.7 we have for smooth nonnegative test functions $\Phi$

$$
E_{h}\left(c_{h}, \kappa\right):=\sum_{t^{n} \in J \in I} \sum_{j} E_{j}^{n}\left(c_{h}, \kappa\right) \frac{1}{\Delta t^{n}} \int_{t^{n}}^{t^{n+1}} \int_{\Omega_{j}} \Phi(x, t) \mathrm{d} x \mathrm{~d} t \leq 0 .
$$

Therefore we have $E\left(c_{h}, \kappa\right) \geq E\left(c_{h}, \kappa\right)+E_{h}\left(c_{h}, \kappa\right)$ and it remains to show a lower bound for $E\left(c_{h}, \kappa\right)+E_{h}\left(c_{h}, \kappa\right)$. From the Definitions 4.6 and 4.8 we get $E\left(c_{h}, \kappa\right)+E_{h}\left(c_{h}, \kappa\right)=T_{t}+T_{c}+T_{d}+T_{\lambda}$, with

$$
\begin{aligned}
T_{t}= & \int_{\mathbb{R}^{2} \times R^{+}} U\left(c_{h}(x, t)-\kappa\right) \partial_{t} \Phi(x, t) \mathrm{d} x \mathrm{~d} t+\int_{\mathbb{R}^{2}} U\left(c_{0}(x)-\kappa\right) \Phi(x, 0) \mathrm{d} x \\
& +\sum_{t^{n} \in J} \sum_{j \in I}\left|\Omega_{j}\right|\left(U\left(c_{j}^{n+1}-\kappa\right)-U\left(c_{j}^{n}-\kappa\right)\right) \Phi_{j}^{n+1}, \\
T_{c}= & \int_{\mathbb{R}^{2} \times R^{+}} F_{U}\left(c_{h}(x, t), \kappa\right) \mathbf{u}(x, t) \cdot \nabla \Phi(x, t) \mathrm{d} x \mathrm{~d} t+\sum_{t^{n} \in J j \in I} \Delta t^{n} \sum_{\substack{l \in N(j), * \in \mathcal{P} M}} G_{j l}^{*, n+1}\left(c_{j}^{n+1}, c_{l}^{n+1}\right) \Phi_{j}^{n+1}, \\
T_{d}= & -\int_{\mathbb{R}^{2} \times R^{+}} D\left(U^{\prime}\left(c_{h}(x, t)-\kappa\right) \nabla c_{h}(x, t) \cdot \nabla \Phi(x, t)+U^{\prime \prime}\left(c_{h}(x, t)-\kappa\right)\left|\nabla c_{h}(x, t)\right|^{2} \Phi(x, t)\right) \mathrm{d} x \mathrm{~d} t \\
& +\sum_{t^{n} \in J j \in I} \sum_{j \in N(j)} \Delta t^{n} D \int_{T_{j l}^{a}} \nabla c_{h}^{n+1} \cdot \nabla N_{j} U^{\prime}\left(c_{j}^{n+1}-\kappa\right) \Phi_{j}^{n+1} \mathrm{~d} x, \\
T_{\lambda}= & -\int_{\mathbb{R}^{2} \times R^{+}} \lambda(x, t) c_{h}(x, t) U^{\prime}\left(c_{h}(x, t)-\kappa\right) \Phi(x, t) \mathrm{d} x \mathrm{~d} t+\sum_{t^{n} \in J j \in I} \sum_{j} \Delta t^{n}\left|\Omega_{j}\right| \lambda_{j}^{n+1} c_{j}^{n+1} U^{\prime}\left(c_{j}^{n+1}-\kappa\right) \Phi_{j}^{n+1},
\end{aligned}
$$

where $\Phi_{j}^{n+1}:=\frac{1}{\left|\Omega_{j}\right| \Delta t^{n}} \int_{t^{n}}^{t^{n+1}} \int_{\Omega_{j}} \Phi(x, t) \mathrm{d} x \mathrm{~d} t$. In order to estimate the Terms $T_{t}, T_{c}, T_{d}$ and $T_{\lambda}$ we first introduce the projection operator $\Pi_{h}$ from $C^{0}\left(\mathbb{R}^{2}\right)$ into the space of piecewise constant functions on the dual cells which is defined as $\left.\Pi_{h}(v)\right|_{\Omega_{j}}=v\left(p_{j}\right)$ for all $j \in I$. For the terms $T_{t}, T_{c}$ and $T_{\lambda}$ we will work with the piecewise constant projection of the approximate solution and apply similar techniques as in the purely hyperbolic situation ( $c f .[10,21,33])$, whereas we exploit the full piecewise linear representation of the approximate solution in order to estimate $T_{d}$.

Estimate of the term $T_{t}$ :

Inserting $\Pi_{h} U\left(c_{h}(x, t)-\kappa\right)$ and using partial summation we get

$$
\begin{aligned}
T_{t}= & -\sum_{t^{n} \in J} \sum_{j \in I} \int_{\Omega_{j}}\left(U\left(c_{j}^{n+1}-\kappa\right)-U\left(c_{j}^{n}-\kappa\right)\right) \Phi\left(x, t^{n}\right) \mathrm{d} x \mathrm{~d} t \\
& -\int_{\mathbb{R}^{2}}\left(\Pi_{h} U\left(c_{h}(x, 0)-\kappa\right)-U\left(c_{0}(x)-\kappa\right)\right) \Phi(x, 0) \mathrm{d} x \\
& +\sum_{t^{n} \in J} \int_{\mathbb{R}^{2}}\left(U\left(c_{h}\left(x, t^{n+1}\right)-\kappa\right)-\Pi_{h} U\left(c_{h}\left(x, t^{n+1}\right)-\kappa\right)\right) \int_{t^{n}}^{t^{n+1}} \partial_{t} \Phi(x, t) \mathrm{d} x \mathrm{~d} t \\
& +\sum_{t^{n} \in J} \sum_{j \in I}\left|\Omega_{j}\right|\left(U\left(c_{j}^{n+1}-\kappa\right)-U\left(c_{j}^{n}-\kappa\right)\right) \Phi_{j}^{n+1} .
\end{aligned}
$$


Estimating $T_{t}$ we get with the properties of $U$ (Def. 4.2)

$$
\begin{aligned}
T_{t} \geq & \sum_{t^{n} \in J j \in I} \sum_{j}\left(U\left(c_{j}^{n+1}-\kappa\right)-U\left(c_{j}^{n}-\kappa\right)\right) \frac{1}{\Delta t^{n}} \int_{t^{n}}^{t^{n+1}} \int_{\Omega_{j}}\left(\Phi(x, t)-\Phi\left(x, t^{n}\right)\right) \mathrm{d} x \mathrm{~d} t \\
& -\int_{\mathbb{R}^{2}}\left|c_{0}(x)-c_{h}(x, 0)\right| \Phi(x, 0) \mathrm{d} x-\sum_{t^{n} \in J} \int_{\mathbb{R}^{2}}\left|c_{h}\left(x, t^{n+1}\right)-\Pi_{h} c_{h}\left(x, t^{n+1}\right)\right| \int_{t^{n}}^{t^{n+1}}\left|\partial_{t} \Phi(x, t)\right| \mathrm{d} x \mathrm{~d} t \\
\geq & -\sum_{t^{n} \in J j \in I} \sum_{j}\left|c_{j}^{n+1}-c_{j}^{n}\right| \int_{t^{n}}^{t^{n+1}} \int_{\Omega_{j}} \int_{0}^{1}\left|\partial_{t} \Phi\left(x, t^{n}+\theta\left(t-t^{n}\right)\right)\right| \mathrm{d} \theta \mathrm{d} x \mathrm{~d} t \\
& -\int_{\mathbb{R}^{2}}\left|c_{0}(x)-c_{h}(x, 0)\right| \Phi(x, 0) \mathrm{d} x-\sum_{t^{n} \in J j \in I} \sum_{j} h_{\Omega_{j}}\left|\nabla c_{h}\left(x, t^{n+1}\right)\right| \int_{t^{n}}^{t^{n+1}}\left|\partial_{t} \Phi(x, t)\right| \mathrm{d} x \mathrm{~d} t .
\end{aligned}
$$

Estimate of the term $T_{c}$ :

To estimate this term we add and subtract $\Pi_{h} F_{U}\left(c_{h}(x, t), \kappa\right)$ into the integral and use integration by parts

$$
\begin{aligned}
T_{c}= & \sum_{t^{n} \in J \in I} \sum_{t^{n}} \int_{\substack{t^{n+1} \\
* \in \mathcal{P} M}}\left(F_{U}\left(c_{j}^{n+1}, \kappa\right) \int_{S_{j l}^{*}} \mathbf{u}(\gamma, t) \cdot \mathbf{n}_{j l}^{*} \Phi(\gamma, t) \mathrm{d} \gamma+\frac{1}{\left|\Omega_{j}\right|} G_{j l}^{*, n+1}\left(c_{j}^{n+1}, c_{l}^{n+1}\right) \int_{\Omega_{j}} \Phi(x, t) \mathrm{d} x\right) \mathrm{d} t \\
& +\int_{\mathbb{R}^{2} \times R^{+}}\left(F_{U}\left(c_{h}(x, t), \kappa\right)-\Pi_{h} F_{U}\left(c_{h}(x, t), \kappa\right)\right) \mathbf{u}(x, t) \cdot \nabla \Phi(x, t) \mathrm{d} x \mathrm{~d} t=: T_{c 1}+T_{c 2} .
\end{aligned}
$$

The term $T_{c 1}$ is estimated in the same way as in Theorem 4.1 in [21]. First we get

$$
\begin{aligned}
T_{c 1}= & \sum_{t^{n} \in J(j, l, *) \in \mathcal{E}^{*}}\left(G_{j l}^{*, n+1}\left(c_{j}^{n+1}, c_{l}^{n+1}\right)-G_{j l}^{*, n+1}\left(c_{j}^{n+1}, c_{j}^{n+1}\right)\right) \\
\times & \left(\frac{1}{\left|\Omega_{j}\right|} \int_{t^{n}}^{t^{n+1}} \int_{\Omega_{j}} \Phi(x, t) \mathrm{d} x \mathrm{~d} t-\frac{1}{\left|S_{j l}^{*}\right|} \int_{t^{n}}^{t^{n+1}} \int_{S_{j l}^{*}} \Phi(\gamma, t) \mathrm{d} \gamma \mathrm{d} t\right) \\
+ & \sum_{t^{n} \in J(j, l, *) \in \mathcal{E}^{*}}\left(G_{l j}^{*, n+1}\left(c_{l}^{n+1}, c_{j}^{n+1}\right)-G_{l j}^{*, n+1}\left(c_{l}^{n+1}, c_{l}^{n+1}\right)\right) \\
\times & \left(\frac{1}{\left|\Omega_{l}\right|} \int_{t^{n}}^{t^{n+1}} \int_{\Omega_{l}} \Phi(x, t) \mathrm{d} x \mathrm{~d} t-\frac{1}{\left|S_{j l}^{*}\right|} \int_{t^{n}}^{t^{n+1}} \int_{S_{j l}^{*}} \Phi(\gamma, t) \mathrm{d} \gamma \mathrm{d} t\right) \\
& -\sum_{t^{n} \in J(j, l, *) \in \mathcal{E}^{*}} \int_{t^{n}}^{t^{n+1}} \int_{S_{j l}^{*}}\left(\frac{1}{\left|S_{j l}^{*}\right|}\left(G_{j l}^{*, n+1}\left(c_{j}^{n+1}, c_{j}^{n+1}\right)\right)-F_{U}\left(c_{j}^{n+1}, \kappa\right) \mathbf{u}(\gamma, t) \cdot \mathbf{n}_{j l}^{*}\right) \\
& \left.\times \frac{1}{\Delta t^{n}\left|S_{j l}^{*}\right|} \int_{t^{n}}^{t^{n+1}} \int_{S_{j l}^{*}}(\Phi(\gamma, t)-\Phi(\xi, \tau)) \mathrm{d} \xi \mathrm{d} \tau\right) \mathrm{d} \gamma \mathrm{d} t \\
+ & \sum_{t^{n} \in J(j, l, *) \in \mathcal{E}^{*}} \int_{t^{n}}^{t^{n+1}}\left(\frac{1}{\left|S_{j l}^{*}\right|}\left(G_{j l}^{*, n+1}\left(c_{l}^{n+1}, c_{l}^{n+1}\right)\right)-F_{U}\left(c_{l}^{n+1}, \kappa\right) \mathbf{u}(\gamma, t) \cdot \mathbf{n}_{j l}^{*}\right) \\
& \left.\times \frac{1}{\Delta t^{n}\left|S_{j l}^{*}\right|} \int_{t^{n}}^{t^{n+1}} \int_{S_{j l}^{*}}(\Phi(\gamma, t)-\Phi(\xi, \tau)) \mathrm{d} \xi \mathrm{d} \tau\right) \mathrm{d} \gamma \mathrm{d} t \\
=: & T_{c 1, a}+T_{c 1, b}+T_{c 1, c}+T_{c 1, d} .
\end{aligned}
$$


Using the monotony of the entropy fluxes and $\Phi(x, t)-\Phi(\gamma, t)=\int_{0}^{1} \nabla \Phi(\gamma+\theta(x-\gamma), t)(x-\gamma) \mathrm{d} \theta$, we further derive for the terms $T_{c 1, a}, T_{c 1, b}$

$$
\begin{aligned}
\left|T_{c 1, a}\right| & \leq \sum_{t^{n} \in J(j, l, *) \in \mathcal{E}^{*}} C_{j l}^{*, n+1}\left(c_{j}^{n+1}, c_{l}^{n+1}\right)\left|c_{j}^{n+1}-c_{l}^{n+1}\right|\left(h_{j}+\Delta t^{n}\right) \Delta t^{n}\left\langle\mu_{j l}^{*, n},\left|\partial_{t} \Phi\right|+|\nabla \Phi|\right\rangle, \\
\left|T_{c 1, b}\right| & \leq \sum_{t^{n} \in J(j, l, *) \in \mathcal{E}^{*}} \sum_{l j}^{*, n+1}\left(c_{l}^{n+1}, c_{j}^{n+1}\right)\left|c_{j}^{n+1}-c_{l}^{n+1}\right|\left(h_{l}+\Delta t^{n}\right) \Delta t^{n}\left\langle\mu_{l j}^{*, n},\left|\partial_{t} \Phi\right|+|\nabla \Phi|\right\rangle,
\end{aligned}
$$

where the measures $\mu_{j l}^{*, n}$ are defined in the lemma. To estimate the terms $T_{c 1, c}, T_{c 1, d}$ we use the consistency of the entropy fluxes and get in a similar way

$$
\begin{aligned}
\left|T_{c 1, c}+T_{c 1, d}\right| \leq & \sum_{t^{n} \in J(j, l, *) \in \mathcal{E}^{*}}\left|F_{U}\left(c_{l}^{n+1}, \kappa\right)-F_{U}\left(c_{j}^{n+1}, \kappa\right)\right| \frac{1}{\left(\Delta t^{n}\left|S_{j l}^{*}\right|\right)^{2}} \\
& \times \int_{t^{n}}^{t^{n+1}} \int_{S_{j l}^{*}}\left|\int_{t^{n}}^{t^{n+1}} \int_{S_{j l}^{*}}(\mathbf{u}(\gamma, t)-\mathbf{u}(\xi, \tau)) \cdot \mathbf{n}_{j l}^{*} \mathrm{~d} \xi \mathrm{d} \tau \int_{t^{n}}^{t^{n+1}} \int_{S_{j l}^{*}}(\Phi(\gamma, t)-\Phi(\xi, \tau)) \mathrm{d} \xi \mathrm{d} \tau\right| \mathrm{d} \gamma \mathrm{d} t \\
\leq & \sum_{t^{n} \in J(j, l, *) \in \mathcal{E}^{*}} L_{f}\left|c_{l}^{n+1}-c_{j}^{n+1}\right|\left(\Delta t^{n}+h_{j l}\right)\left\langle\nu_{j l}^{*, n},|\nabla \Phi|+\left|\partial_{t} \Phi\right|\right\rangle,
\end{aligned}
$$

where the measures $\nu_{j l}^{*, n}$ are defined in the lemma and $L_{f}$ denotes the Lipschitz constant of $f$. To estimate the term $T_{c 2}$ we argue as follows

$$
\begin{aligned}
\left|T_{c 2}\right| & =\left|\sum_{t^{n} \in J} \sum_{j \in I} \int_{t^{n}}^{t^{n+1}} \int_{\Omega_{j}}\left(F_{U}\left(c_{h}\left(x, t^{n+1}\right), \kappa\right)-F_{U}\left(c_{j}^{n+1}, \kappa\right)\right) \mathbf{u}(x, t) \cdot \nabla \Phi(x, t) \mathrm{d} x \mathrm{~d} t\right| \\
& \leq \sum_{t^{n} \in J j \in I} \sum_{j \in \mathbf{u}} \|\left.\mathbf{u}\right|_{L^{\infty}\left(\Omega_{j} \times\left(t^{n}, t^{n+1}\right]\right)} L_{f} h_{j} \int_{\Omega_{j}}\left|\nabla c_{h}\left(x, t^{n+1}\right)\right| \int_{t^{n}}^{t^{n+1}}|\nabla \Phi(x, t)| \mathrm{d} x \mathrm{~d} t
\end{aligned}
$$

where $L_{f}$ is the Lipschitz constant of the flux $f$.

Estimate of the term $T_{d}$ :

To estimate the term $T_{d}$ we first write all terms as a summation over triangles $T_{k} \in \mathcal{T}$

$$
\begin{aligned}
T_{d}= & -\sum_{t^{n} \in J} \sum_{k \in K} \int_{t^{n}}^{t^{n+1}} \int_{T_{k}} D U^{\prime}\left(c_{h}(x, t)-\kappa\right) \nabla c_{h}(x, t) \cdot \nabla \Phi(x, t) \mathrm{d} x \mathrm{~d} t \\
& -\sum_{t^{n} \in J} \sum_{k \in K} \int_{t^{n}}^{t^{n+1}} \int_{T_{k}} D U^{\prime \prime}\left(c_{h}(x, t)-\kappa\right)\left|\nabla c_{h}(x, t)\right|^{2} \Phi(x, t) \mathrm{d} x \mathrm{~d} t \\
& +\sum_{t^{n} \in J} \sum_{k \in K} \int_{t^{n}}^{t^{n+1}} \int_{T_{k}} D \nabla c_{h}^{n+1} \cdot \nabla \Pi_{h}^{1}\left(U^{\prime}\left(c_{h}^{n+1}-\kappa\right) \Pi_{h}^{0}(\Phi)\right) \mathrm{d} x \mathrm{~d} t .
\end{aligned}
$$

Here $\Pi_{h}^{1}$ denotes the nodal projection operator into $V_{h}$ (e.g. if $\left\{N_{l}\right\}_{l}$ denote the linear base functions of $V_{h}$ we have $\left.\left.\Pi_{h}^{1}(v)\right|_{T_{k}}=\sum_{\left\{l \mid p_{l} \in T_{k}\right\}} v\left(p_{l}\right) N_{l}\right)$ and the projection $\Pi_{h}^{0}$ is defined by $\Pi_{h}^{0}(\Phi)(x, t):=\Phi_{j}^{n+1}$ for $x \in \Omega_{j}$ and 
$t \in\left(t^{n}, t^{n+1}\right]$. We now use integration by parts on the triangles $T_{k}$ in the first and third term to get

$$
\begin{aligned}
T_{d}= & \sum_{t^{n} \in J k \in K} \sum_{t^{n}} \int_{T_{k}}^{t^{n+1}} \int_{T^{\prime}} D \nabla\left(U^{\prime}\left(c_{h}(x, t)-\kappa\right) \nabla c_{h}(x, t)\right) \Phi(x, t) \mathrm{d} x \mathrm{~d} t \\
& -\sum_{t^{n} \in J k \in K} \sum_{t^{n}} \int_{T_{k}}^{t^{n+1}} D U^{\prime \prime}\left(c_{h}(x, t)-\kappa\right)\left|\nabla c_{h}(x, t)\right|^{2} \Phi(x, t) \mathrm{d} x \mathrm{~d} t \\
& -\left.\sum_{t^{n} \in J} \sum_{j \in I} \sum_{l \in N(j)} \int_{t^{n}}^{t^{n+1}} \int_{\Gamma_{j l}} D \nabla c_{h}^{n+1}\right|_{T_{j l}^{a}} \cdot \mathbf{m}_{j l} U^{\prime}\left(c_{h}(\gamma, t)-\kappa\right) \Phi(\gamma, t) \mathrm{d} \gamma \mathrm{d} t \\
& +\left.\sum_{t^{n} \in J} \sum_{j \in I} \sum_{l \in N(j)} \int_{t^{n}}^{t^{n+1}} \int_{\Gamma_{j l}} D \nabla c_{h}^{n+1}\right|_{T_{j l}^{a}} \cdot \mathbf{m}_{j l} \Pi_{h}^{1}\left(U^{\prime}\left(c_{h}^{n+1}-\kappa\right) \Pi_{h}^{0}(\Phi)\right) \mathrm{d} \gamma \mathrm{d} t .
\end{aligned}
$$

As $\left.\Delta c_{h}^{n+1}\right|_{\Omega_{j} \cap T_{j l}^{a}}=0$ we see that the first two terms of $T_{d}$ cancel. For the remaining terms we now write the sums over $j$ and $l$ as a sum over the edges $(j, l)$ and use $T_{l j}^{a}=T_{j l}^{b}$ as well as $\mathbf{m}_{j l}=-\mathbf{m}_{l j}$. This yields

$$
\left|T_{d}\right| \leq \sum_{t^{n} \in J(j, l) \in \mathcal{E}} \int_{t^{n}}^{t^{n+1}} \int_{\Gamma_{j l}} D\left[\nabla c_{h}^{n+1} \cdot \mathbf{m}_{j l}\right]_{\Gamma_{j l}}\left|\Pi_{h}^{1}\left(U^{\prime}\left(c_{h}^{n+1}-\kappa\right) \Pi_{h}^{0}(\Phi)\right)-U^{\prime}\left(c_{h}(\gamma, t)-\kappa\right) \Phi(\gamma, t)\right| \mathrm{d} \gamma \mathrm{d} t,
$$

where we used the notation $\left[\nabla c_{h}^{n+1} \cdot \mathbf{m}_{j l}\right]_{\Gamma_{j l}}:=\left|\left(\left.\nabla c_{h}^{n+1}\right|_{T_{j l}^{a}}-\left.\nabla c_{h}^{n+1}\right|_{T_{j l}^{b}}\right) \cdot \mathbf{m}_{j l}\right|$. Now it remains to estimate

$$
I:=\int_{t^{n}}^{t^{n+1}} \int_{\Gamma_{j l}} \Pi_{h}^{1}\left(U^{\prime}\left(c_{h}^{n+1}-\kappa\right) \Pi_{h}^{0}(\Phi)\right)-U^{\prime}\left(c_{h}(\gamma, t)-\kappa\right) \Phi(\gamma, t) \mathrm{d} \gamma \mathrm{d} t
$$

which can be done as follows

$$
\begin{aligned}
I= & \int_{t^{n}}^{t^{n+1}} \int_{\Gamma_{j l}} N_{j} U^{\prime}\left(c_{j}^{n+1}-\kappa\right) \Phi_{j}^{n+1}+N_{l} U^{\prime}\left(c_{l}^{n+1}-\kappa\right) \Phi_{l}^{n+1}-U^{\prime}\left(c_{h}(\gamma, t)-\kappa\right) \Phi(\gamma, t) \mathrm{d} \gamma \mathrm{d} t \\
= & \int_{t^{n}}^{t^{n+1}} \int_{\Gamma_{j l}} N_{j} U^{\prime}\left(c_{j}^{n+1}-\kappa\right)\left(\Phi_{j}^{n+1}-\Phi(\gamma, t)\right)+N_{l} U^{\prime}\left(c_{l}^{n+1}-\kappa\right)\left(\Phi_{l}^{n+1}-\Phi(\gamma, t)\right) \mathrm{d} \gamma \mathrm{d} t \\
& +\int_{t^{n}}^{t^{n+1}} \int_{\Gamma_{j l}}\left(N_{j}\left(U^{\prime}\left(c_{j}^{n+1}-\kappa\right)-U^{\prime}\left(c_{h}(\gamma, t)-\kappa\right)\right)+N_{l}\left(U^{\prime}\left(c_{l}^{n+1}-\kappa\right)-U^{\prime}\left(c_{h}(\gamma, t)-\kappa\right)\right)\right) \Phi(\gamma, t) \mathrm{d} \gamma \mathrm{d} t . \\
|I| \leq & \Delta t^{n}\left|\Gamma_{j l}\right|\left(h_{j l}^{n}+\Delta t^{n}\right)\left(\left\langle\mu_{j l}^{n},\left|\partial_{t} \Phi\right|+|\nabla \Phi|\right\rangle+\left\langle\mu_{l j}^{n},\left|\partial_{t} \Phi\right|+|\nabla \Phi|\right\rangle\right) \\
& +\frac{2}{\delta}|| \bar{U}^{\prime \prime} \|_{L^{\infty}(\mathbb{R})}\left|c_{j}^{n+1}-c_{l}^{n+1}\right| \int_{t^{n}}^{t^{n+1}} \int_{\Gamma_{j l}} \Phi(\gamma, t) \mathrm{d} \gamma \mathrm{d} t,
\end{aligned}
$$

where the measure $\mu_{j l}^{n}$ is defined as in the statement of the Lemma. With this estimate on $I$ we finally get the following estimate on $T_{d}$

$$
\begin{aligned}
\left|T_{d}\right| \leq & \sum_{t^{n} \in J(j, l) \in \mathcal{E}} D\left[\nabla c_{h}^{n+1} \cdot \mathbf{m}_{j l}\right]_{\Gamma_{j l}}\left(\frac{2}{\delta}|| \bar{U}^{\prime \prime}||_{L^{\infty}(\mathbb{R})}\left|c_{j}^{n+1}-c_{l}^{n+1}\right| \int_{t^{n}}^{t^{n+1}} \int_{\Gamma_{j l}} \Phi(\gamma, t) \mathrm{d} \gamma \mathrm{d} t\right. \\
& \left.+\Delta t^{n}\left|\Gamma_{j l}\right|\left(h_{j l}^{n}+\Delta t^{n}\right)\left(\left\langle\mu_{j l}^{n},\left|\partial_{t} \Phi\right|+|\nabla \Phi|\right\rangle+\left\langle\mu_{l j}^{n},\left|\partial_{t} \Phi\right|+|\nabla \Phi|\right\rangle\right)\right) .
\end{aligned}
$$


Estimate of the term $T_{\lambda}$ :

We again introduce a projection term $\Pi_{h} U^{\prime}\left(c_{h}(x, t)-\kappa\right)$ and get

$$
\begin{aligned}
& T_{\lambda}=\sum_{t^{n} \in J \in I} \sum_{j}^{n+1} U^{\prime}\left(c_{j}^{n+1}-\kappa\right) \int_{t^{n}}^{t^{n+1}} \int_{\Omega_{j}} \Phi(x, t)\left(\lambda_{j}^{n+1}-\lambda(x, t)\right) \mathrm{d} x \mathrm{~d} t \\
& +\int_{\mathbb{R}^{2} \times R^{+}} \lambda(x, t)\left(\Pi_{h}\left(c_{h}(x, t) U^{\prime}\left(c_{h}(x, t)-\kappa\right)\right)-c_{h}(x, t) U^{\prime}\left(c_{h}(x, t)-\kappa\right)\right) \Phi(x, t) \mathrm{d} x \mathrm{~d} t \\
& \leq \sum_{t^{n} \in J} \sum_{j \in I}\left|c_{j}^{n+1}\right| \int_{t^{n}}^{t^{n+1}} \int_{\Omega_{j}} \Phi(x, t)\left|\lambda_{j}^{n+1}-\lambda(x, t)\right| \mathrm{d} x \mathrm{~d} t \\
& +\sum_{t^{n} \in J} \sum_{j \in I} \int_{t^{n}}^{t^{n+1}} \int_{\Omega_{j}} \lambda(x, t)\left(c_{j}^{n+1} U^{\prime}\left(c_{j}^{n+1}-\kappa\right)-c_{h}\left(x, t^{n+1}\right) U^{\prime}\left(c_{h}\left(x, t^{n+1}\right)-\kappa\right)\right) \Phi(x, t) \mathrm{d} x \mathrm{~d} t \\
& \leq \sum_{t^{n} \in J} \sum_{j \in I}\left|c_{j}^{n+1}\right| \int_{t^{n}}^{t^{n+1}} \int_{\Omega_{j}} \Phi(x, t)\left|\lambda_{j}^{n+1}-\lambda(x, t)\right| \mathrm{d} x \mathrm{~d} t \\
& +\sum_{t^{n} \in J j \in I} \sum_{j}\|\lambda\|_{L^{\infty}\left(\Omega_{j} \times\left(t^{n}, t^{n+1}\right]\right)} \int_{t^{n}}^{t^{n+1}} \int_{\Omega_{j}}\left|c_{j}^{n+1}-c_{h}\left(x, t^{n+1}\right)\right| \Phi(x, t) \mathrm{d} x \mathrm{~d} t \\
& +\sum_{t^{n} \in J j \in I} \sum_{j \in \lambda}\left\|_{L^{\infty}\left(\Omega_{j} \times\left(t^{n}, t^{n+1}\right]\right)} \int_{t^{n}}^{t^{n+1}} \int_{\Omega_{j}}\left|c_{j}^{n+1}\right| \frac{1}{\delta}\right\| \bar{U}^{\prime \prime} \|_{L^{\infty}(\mathbb{R})}\left|c_{j}^{n+1}-c_{h}\left(x, t^{n+1}\right)\right| \Phi(x, t) \mathrm{d} x \mathrm{~d} t \\
& \leq \sum_{t^{n} \in J j \in I}\left|c_{j}^{n+1}\right| \int_{t^{n}}^{t^{n+1}} \int_{\Omega_{j}} \Phi(x, t)\left|\lambda_{j}^{n+1}-\lambda(x, t)\right| \mathrm{d} x \mathrm{~d} t \\
& +\sum_{t^{n} \in J} \sum_{j \in I}\|\lambda\|_{L^{\infty}\left(\Omega_{j} \times\left(t^{n}, t^{n+1}\right]\right)}\left(1+\left|c_{j}^{n+1}\right| \frac{1}{\delta}\left\|\bar{U}^{\prime \prime}\right\|_{L^{\infty}(\mathbb{R})}\right) h_{j} \int_{\Omega_{j}}\left|\nabla c_{h}\left(x, t^{n+1}\right)\right| \int_{t^{n}}^{t^{n+1}} \Phi(x, t) \mathrm{d} t \mathrm{~d} x .
\end{aligned}
$$

Collecting all the estimates shown above, we have proved the Lemma.

Definition 4.10 (Choice of the test function). Define the functions $w_{0} \in C_{0}^{\infty}(\mathbb{R}, \mathbb{R})$ and $\bar{w}_{0} \in C_{0}^{\infty}\left(\mathbb{R}^{2}, \mathbb{R}\right)$ as $\operatorname{supp}\left(w_{0}\right) \subset[-1,0], w_{0} \geq 0, \int_{\mathbb{R}} w_{0}(x) \mathrm{d} x=1, \operatorname{supp}\left(\bar{w}_{0}\right) \subset\left\{x \in \mathbb{R}^{2}|| x \mid \leq 1\right\}, \bar{w}_{0} \geq 0, \int_{\mathbb{R}^{2}} \bar{w}_{0}(x) \mathrm{d} x=1$. Furthermore for all $r \geq 1$ let us define $w, \bar{w}$ as

$$
w(t)=r w_{0}(r t), \quad \bar{w}(x)=r^{d} \bar{w}_{0}(r x) .
$$

Let $\psi(t):=\mathrm{e}^{M(T-t)}-1, \forall t \in[0, T]$, where $M:=\|\lambda\|_{L^{\infty}\left((0, T) ; \mathbb{R}^{2}\right)}$ then we set:

$$
\Phi(x, t)=\varphi(x, t, y, s):=\psi(t) \bar{w}(x-y) w(t-s) .
$$

With this choice of the test function we are now able to state the main result of this section which is the continuous entropy estimate of the approximate solution.

Corollary 4.11. Let $c_{h}$ be the discrete solution defined in 3.5 and let $c$ be the entropy solution of (1). Furthermore, let the Assumptions 2.7 and 3.1 be fulfilled and let $\Phi(x, t)$ be defined as in (23). Then we get the 
following estimate:

$$
\begin{aligned}
\bar{E}\left(c_{h}, c\right):=\int_{\mathbb{R}^{2} \times R^{+}} E\left(c_{h}, c(y, s)\right) \mathrm{d} y \mathrm{~d} s \geq & -\bar{R}_{h}\left(\eta_{0}, \eta_{t}, \eta_{c}, \eta_{d}, \eta_{\lambda}, \eta_{p, 0}, \eta_{p, 1}, \eta_{U}\right) \\
\bar{R}_{h}\left(\eta_{0}, \eta_{t}, \eta_{c}, \eta_{d}, \eta_{\lambda}, \eta_{p, 0}, \eta_{p, 1}, \eta_{U}\right):= & \left(\eta_{0}+\eta_{p, 0}+\eta_{\lambda}\right)+\frac{1}{\delta} \eta_{U}+\left\|\psi_{t}\right\|_{L^{\infty}(0, T)}\left(\eta_{t}+\eta_{c}+\eta_{d}+\eta_{p, 1}\right) \\
& +r\|\psi\|_{L^{\infty}(0, T)}\left(\left(K_{1}+K_{1}^{\prime}\right)\left(\eta_{t}+\eta_{c}+\eta_{d}+\eta_{p, 1}\right)\right)
\end{aligned}
$$

and

$$
\begin{aligned}
& \eta_{0}=\int_{\mathbb{R}^{2}}\left|c_{h}(x, 0)-c_{0}(x)\right| \mathrm{d} x, \\
& \eta_{t}=\sum_{t^{n} \in J} \sum_{j \in I}\left|c_{j}^{n+1}-c_{j}^{n}\right| \Delta t^{n}\left|\Omega_{j}\right| \\
& \left.\eta_{c}=\sum_{t^{n} \in J(j, l, *) \in \mathcal{E}^{*}} \sum_{j l}+\Delta t^{n}\right) \Delta t^{n} Q_{j l}^{*, n+1}\left(c_{j}^{n+1}, c_{l}^{n+1}\right)\left|c_{j}^{n+1}-c_{l}^{n+1}\right| \\
& +\sum_{t^{n} \in J(j, l, *) \in \mathcal{E}^{*}} \sum_{f}|\|\mathbf{u}\||_{\left(S_{j l}^{*} \times\left(t^{n}, t^{n+1}\right)\right)}\left|c_{j}^{n+1}-c_{l}^{n+1}\right|\left(h_{j l}+\Delta t^{n}\right)^{2} \Delta t^{n}\left|S_{j l}^{*}\right|,
\end{aligned}
$$

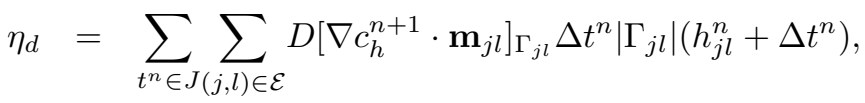

$$
\begin{aligned}
& \eta_{\lambda}=\sum_{t^{n} \in J} \sum_{j \in I}\left|c_{j}^{n+1}\right| \int_{t^{n}}^{t^{n+1}} \int_{\Omega_{j}}\left|\lambda_{j}^{n+1}-\lambda(x, t)\right| \mathrm{d} x \mathrm{~d} t, \\
& \eta_{p, 0}=\sum_{t^{n} \in J} \sum_{j \in I}\|\lambda\|_{L^{\infty}\left(\Omega_{j} \times\left(t^{n}, t^{n+1}\right]\right)} h_{j} \Delta t^{n} \int_{\Omega_{j}}\left|\nabla c_{h}\left(x, t^{n+1}\right)\right| \mathrm{d} x, \\
& \eta_{p, 1}=\sum_{t^{n} \in J} \sum_{j \in I}\left(1+\|\mathbf{u}\|_{L^{\infty}\left(\Omega_{j} \times\left(t^{n}, t^{n+1}\right]\right)} L_{f}\right) h_{j} \Delta t^{n} \int_{\Omega_{j}}\left|\nabla c_{h}\left(x, t^{n+1}\right)\right| \mathrm{d} x, \\
& \eta_{U}=\left\|\bar{U}^{\prime \prime}\right\|_{L^{\infty}(\mathbb{R})} \sum_{t^{n} \in J} \sum_{j \in I}\left(\|\lambda\|_{L^{\infty}\left(\Omega_{j} \times\left(t^{n}, t^{n+1}\right]\right)}\left|c_{j}^{n+1}\right| h_{j} \Delta t^{n} \int_{\Omega_{j}}\left|\nabla c_{h}\left(x, t^{n+1}\right)\right| \mathrm{d} x\right)
\end{aligned}
$$

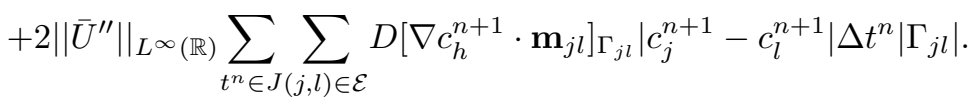

Here $\delta$ is the regularization parameter of the entropy $U$ (cf. Def. 4.2 for the definition of $U, \delta$ and $\bar{U})$, $K_{1}:=\int_{\mathbb{R}}\left|\partial_{t} w_{0}(t)\right| \mathrm{d} t, K_{1}^{\prime}:=\int_{\mathbb{R}^{2}}\left|\nabla \bar{w}_{0}(x)\right| \mathrm{d} x$ and $Q_{j l}^{*, n+1},\left|\|\mathbf{u} \mid\|_{\left(S_{j l}^{*} \times\left(t^{n}, t^{n+1}\right)\right)}\right.$ are defined as

$$
Q_{j l}^{*, n+1}(v, w):=\frac{2 g_{j l}^{*, n+1}(v, w)-g_{j l}^{*, n+1}(v, v)-g_{j l}^{*, n+1}(w, w)}{(v-w)}
$$

$$
\||\mathbf{u}|\|_{\left(S_{j l}^{*} \times\left(t^{n}, t^{n+1}\right)\right)}:=\frac{1}{\left(\Delta t^{n}\left|S_{j l}^{*}\right|\right)^{2}\left(h_{j l}+\Delta t^{n}\right)} \int_{t^{n}}^{t^{n+1}} \int_{S_{j l}^{*}} \int_{t^{n}}^{t^{n+1}} \int_{S_{j l}^{*}}\left|(\mathbf{u}(\gamma, t)-\mathbf{u}(\xi, \tau)) \cdot \mathbf{n}_{j l}^{*}\right| \mathrm{d} \xi \mathrm{d} \tau \mathrm{d} \gamma \mathrm{d} t
$$

Proof. The proof follows analogue to the proof of Corollary 1 in [41]. 


\section{Approximation inequality And a POSTERIORI ERRor estimate}

In the last section we proved, that the approximate solution $c_{h}$ of (1) fulfills an entropy inequality up to an residuum term $\bar{R}_{h}$ (Cor. 4.11). The next step in order to prove an a posteriori error estimate for the finite volume approximation, is an approximation inequality which will be stated in Theorem 5.3 below. Before we can state this result we introduce the following notations.

Definition 5.1 (The two-forms $\bar{E}$ and its dual $\left.\bar{E}^{*}\right)$. For any $\tilde{c}, c \in L^{\infty}\left(0, T ; W^{1,1}\left(\mathbb{R}^{2}\right)\right)$ let the two-form $\bar{E}$ be defined as in Corollary 4.11, equation (24). Furthermore, let us define the two-form $\bar{E}^{*}$ by:

$$
\begin{aligned}
\bar{E}^{*}(\tilde{c}, c):= & \int_{\left(\mathbb{R}^{2} \times R^{+}\right)^{2}} U(c(y, s)-\tilde{c}(x, t)) \partial_{s} \varphi(x, t, y, s) \mathrm{d} x \mathrm{~d} t \mathrm{~d} y \mathrm{~d} s \\
& +\int_{\mathbb{R}^{2} \times R^{+}} \int_{\mathbb{R}^{2}} U\left(c_{0}(y)-\tilde{c}(x, t)\right) \varphi(x, t, y, 0) \mathrm{d} y \mathrm{~d} x \mathrm{~d} t \\
& +\int_{\left(\mathbb{R}^{2} \times R^{+}\right)^{2}} F_{U}(c(y, s), \tilde{c}(x, t)) \mathbf{u}(y, s) \cdot \nabla_{y} \varphi(x, t, y, s) \mathrm{d} x \mathrm{~d} t \mathrm{~d} y \mathrm{~d} s \\
& -\int_{\left(\mathbb{R}^{2} \times R^{+}\right)^{2}} D U^{\prime}(c(y, s)-\tilde{c}(x, t)) \nabla_{y} c(y, s) \cdot \nabla_{y} \varphi(x, t, y, s) \mathrm{d} x \mathrm{~d} t \mathrm{~d} y \mathrm{~d} s \\
& -\int_{\left(\mathbb{R}^{2} \times R^{+}\right)^{2}} D U^{\prime \prime}(c(y, s)-\tilde{c}(x, t))\left|\nabla_{y} c(y, s)\right|^{2} \varphi(x, t, y, s) \mathrm{d} x \mathrm{~d} t \mathrm{~d} y \mathrm{~d} s \\
& -\int_{\left(\mathbb{R}^{2} \times R^{+}\right)^{2}} \lambda(y, s) c(y, s) U^{\prime}(c(y, s)-\tilde{c}(x, t)) \varphi(x, t, y, s) \mathrm{d} x \mathrm{~d} t \mathrm{~d} y \mathrm{~d} s .
\end{aligned}
$$

Lemma 5.2. For $\tilde{c}, c \in L^{\infty}\left(0, T ; W^{1,1}\left(\mathbb{R}^{2}\right)\right)$ let $A(\tilde{c}, c)$ be defined as

$$
A(\tilde{c}, c):=\int_{\mathbb{R}^{2} \times R^{+}}|\tilde{c}(x, t)-c(x, t)| \partial_{t} \psi(t) \mathrm{d} x \mathrm{~d} t+\int_{\mathbb{R}^{2} \times R^{+}}|\lambda(x, t)||\tilde{c}(x, t)-c(x, t)| \psi(t) \mathrm{d} x \mathrm{~d} t .
$$

Choose $\psi$ as in Definition 4.10, then the following inequality holds for any $M$ such that $\|\lambda\|_{L^{\infty}\left(\mathbb{R}^{2} \times(0, T)\right)} \leq M$ :

$$
\int_{\mathbb{R}^{2} \times R^{+}}|\tilde{c}(x, t)-c(x, t)| \mathrm{d} x \mathrm{~d} t \leq-\frac{1}{M} A(\tilde{c}, c) .
$$

Proof. Using the definition of $\psi$ and the assumption on $M$ we get the result

$A(\tilde{c}, c) \leq \int_{\mathbb{R}^{2} \times R^{+}}|\tilde{c}(x, t)-c(x, t)| M\left(-\mathrm{e}^{M(T-t)}+\left(\mathrm{e}^{M(T-t)}-1\right)\right) \mathrm{d} x \mathrm{~d} t=-M \int_{\mathbb{R}^{2} \times R^{+}}|\tilde{c}(x, t)-c(x, t)| \mathrm{d} x \mathrm{~d} t$.

In the next step we prove an approximation inequality. Within this proof we use some essential ideas of Cockburn, Gripenberg [15] and Carrillo [8] to handle the diffusive part of the differential equation. Similar results for scalar hyperbolic conservation laws where for example proved in [7,14] and [21].

Theorem 5.3 (Approximation inequality). Let $\tilde{c} \in L^{\infty}\left(0, T ; W^{1,1}\left(\mathbb{R}^{2}\right)\right)$ and let $c$ be the entropy solution of problem (1), where the Assumption 2.7 is supposed to be fulfilled (especially we have $\left.\bar{E}^{*}(\tilde{c}, c)=0\right)$. Then the following approximation inequality holds for any $M>0$ such that $\|\lambda\|_{L^{\infty}\left(\mathbb{R}^{2} \times(0, T)\right)} \leq M$ :

$$
\|\tilde{c}-c\|_{L^{1}\left((0, T) \times \mathbb{R}^{2}\right)}+\frac{1}{M} H(\tilde{c}-c) \leq \frac{1}{M}\left(k_{3} \frac{1}{r}+k_{4} \delta-\bar{E}(\tilde{c}, c)\right),
$$


where $k_{3}$ and $k_{4}$ are given by

$$
\begin{aligned}
k_{3}:= & |\psi(0)|\left(\left(1+K_{3}\right)\left|c_{0}\right|_{B V\left(\mathbb{R}^{2}\right)}\right)+\|\psi\|_{L^{\infty}((0, T))}|| f^{\prime} \|_{L^{\infty}(\mathbb{R})}|\mathbf{u}|_{B V\left(\mathbb{R}^{2} \times(0, T)\right)} \int_{0}^{T}|c(t)|_{B V\left(\mathbb{R}^{2}\right)} \mathrm{d} t \\
& +\|\psi\|_{L^{\infty}((0, T))}\left(\left.\left\|\left.c\right|_{L^{\infty}\left(\mathbb{R}^{2} \times(0, T)\right)}|\lambda|_{B V\left(\mathbb{R}^{2} \times(0, T)\right)}+\right\| \lambda\right|_{L^{\infty}\left(\mathbb{R}^{2} \times(0, T)\right)}|c|_{B V\left(\mathbb{R}^{2} \times(0, T)\right)}\right) \\
k_{4}:= & \|\psi\|_{L^{\infty}((0, T))}\left\|\left.\mathbf{u}\right|_{L^{\infty}\left(\mathbb{R}^{2} \times(0, T)\right)} \frac{k_{1}}{2}\right\| f^{\prime \prime} \|_{L^{\infty}(\mathbb{R})} \int_{0}^{T}|c(t)|_{\left.B V\left(\mathbb{R}^{2}\right)\right)} \mathrm{d} t
\end{aligned}
$$

and $H(\tilde{c}-c)$ is defined as

$$
H(\tilde{c}-c):=\int_{\left(\mathbb{R}^{2} \times R^{+}\right)^{2}} D U^{\prime \prime}(\tilde{c}(x, t)-c(y, s))\left|\nabla_{x} \tilde{c}(x, t)-\nabla_{y} c(y, s)\right|^{2} \psi(t) \bar{w}(x-y) w(t-s) \mathrm{d} x \mathrm{~d} t \mathrm{~d} y \mathrm{~d} s
$$

Furthermore, $K_{3}$ is the constant defined in Theorem 2.9 and $k_{1}$ is defined in Lemma 4.3. Note that $H(\tilde{c}-c)$ is positive for all values $\tilde{c}$ and $c$. Here $|\mathbf{u}|_{B V\left(\mathbb{R}^{2} \times(0, T)\right)}$ is defined as

$$
|\mathbf{u}|_{B V\left(\mathbb{R}^{2} \times(0, T)\right)}:=\sup _{\left\{(\xi, \tau) \in \mathbb{R}^{2} \times \mathbb{R}^{+}|| \xi \mid \leq \frac{1}{r}, \tau \leq \frac{1}{r}\right\}} \int_{\mathbb{R}^{2} \times(0, T)}|\mathbf{u}(x, t)-\mathbf{u}(x+\xi, t+\tau)| r \mathrm{~d} x \mathrm{~d} t .
$$

Proof. From the assumption on $c$ and Lemma 5.2 we get

$$
\int_{\mathbb{R}^{2} \times R^{+}}|\tilde{c}(x, t)-c(x, t)| \mathrm{d} x \mathrm{~d} t \leq \frac{1}{M}\left(\bar{E}(\tilde{c}, c)+\bar{E}^{*}(\tilde{c}, c)-A(\tilde{c}, c)-\bar{E}(\tilde{c}, c)\right) .
$$

Thus, it remains to estimate the term $\bar{E}(\tilde{c}, c)+\bar{E}^{*}(\tilde{c}, c)-A(\tilde{c}, c)$. With the definition of $A, \bar{E}, \bar{E}^{*}$ and using $\varphi(x, y, s, t)=\psi(t) \bar{w}(x-y) w(t-s)\left(c f\right.$. Def. 4.10) we get $\left(\bar{E}+\bar{E}^{*}-A\right)(\tilde{c}, c) \leq I_{0}+I_{t}+I_{c}+I_{d}+I_{\lambda}$, with

$$
\begin{aligned}
I_{0}:= & \int_{\mathbb{R}^{2} \times R^{+}} \int_{\mathbb{R}^{2}} U\left(c_{0}(x)-c(y, s)\right) \psi(0) \bar{w}(x-y) w(-s) \mathrm{d} x \mathrm{~d} y \mathrm{~d} s, \\
I_{t}:= & \int_{\left(\mathbb{R}^{2} \times R^{+}\right)^{2}}(U(\tilde{c}(x, t)-c(y, s))-|\tilde{c}(x, t)-c(x, t)|) \partial_{t} \psi(t) \bar{w}(x-y) w(t-s) \mathrm{d} x \mathrm{~d} t \mathrm{~d} y \mathrm{~d} s, \\
I_{c}:= & -\int_{\left(\mathbb{R}^{2} \times R^{+}\right)^{2}}\left(F_{U}(\tilde{c}(x, t), c(y, s)) \mathbf{u}(x, t)-F_{U}(c(y, s), \tilde{c}(x, t)) \mathbf{u}(y, s)\right) \\
I_{d}:= & \int_{\left(\mathbb{R}^{2} \times R^{+}\right)^{2}} D\left(\nabla_{y} c(y, s) \cdot \nabla_{x} \bar{w}(x-y)-\nabla_{x} \tilde{c}(x, t) \cdot \nabla_{y} \bar{w}(x-y)\right) \\
& -\int_{\left(\mathbb{R}^{2} \times R^{+}\right)^{2}} D U^{\prime \prime}(\tilde{c}(x, t)-c(y, s))\left(\left|\nabla_{x} \tilde{c}(x, t)\right|^{2}+\left|\nabla_{y} c(y, s)\right|^{2}\right) \\
I_{\lambda}:= & \int_{\left(\mathbb{R}^{2} \times R^{+}\right)^{2}}(\lambda(y, s) c(y, s)-\lambda(x, t) \tilde{c}(x, t)) U^{\prime}(\tilde{c}(x, t)-c(y, s)) \psi(t) \bar{w}(x-y) w(t-s) \mathrm{d} x \mathrm{~d} t \mathrm{~d} y \mathrm{~d} s \\
& -\int_{\left(\mathbb{R}^{2} \times R^{+}\right)^{2}}|\lambda(x, t)||\tilde{c}(x, t)-c(x, t)| \psi(t) \bar{w}(x-y) w(t-s) \mathrm{d} x \mathrm{~d} t \mathrm{~d} y \mathrm{~d} s .
\end{aligned}
$$


In what follows these terms will be estimated separately:

The term $I_{0}$ : To estimate the term $I_{0}$ we need a result that the exact solution $c(\cdot, s)$ approaches the initial values $c_{0}$ at least Lipschitz if $s$ goes to zero. This result is stated in Theorem 2.9. Therefore, we have

$$
\begin{aligned}
\left|I_{0}\right| & \leq \int_{\mathbb{R}^{2} \times R^{+}} \int_{\mathbb{R}^{2}}\left(|c(x, 0)-c(y, 0)|+\left|c_{0}(y, s)-c(y, 0)\right|\right) \psi(0) \bar{w}(x-y) w(-s) \mathrm{d} x \mathrm{~d} y \mathrm{~d} s \\
& \leq|\psi(0)|\left(\left(1+K_{3}\right)\left|c_{0}\right|_{B V\left(\mathbb{R}^{2}\right)}\right) \frac{1}{r},
\end{aligned}
$$

where $K_{3}$ is the constant of Theorem 2.9 which is independent of $D$.

\section{The term $I_{t}$ :}

$$
\left|I_{t}\right| \leq \int_{\left(\mathbb{R}^{2} \times R^{+}\right)^{2}}|c(y, s)-c(x, t)| \partial_{t} \psi(t) \bar{w}(x-y) w(t-s) \mathrm{d} x \mathrm{~d} t \mathrm{~d} y \mathrm{~d} s \leq\left\|\partial_{t} \psi\right\|_{L^{\infty}\left(\mathbb{R}^{2} \times(0, T)\right)}|c|_{B V\left(\mathbb{R}^{2} \times(0, T)\right)} \frac{1}{r} .
$$

The term $I_{c}$ : Using integration by parts with respect to $y$ and $\nabla \cdot u=0$ we get

$$
\begin{aligned}
I_{c}= & \int_{\left(\mathbb{R}^{2} \times R^{+}\right)^{2}} \partial_{c}\left(F_{U}(c(y, s), \tilde{c}(x, t)) \mathbf{u}(y, s)-F_{U}(\tilde{c}(x, t), c(y, s)) \mathbf{u}(x, t)\right) \nabla_{y} c(y, s) \\
\times \psi(t) \bar{w}(x-y) w(t-s) \mathrm{d} x \mathrm{~d} t \mathrm{~d} y \mathrm{~d} s & \\
= & \int_{\left(\mathbb{R}^{2} \times R^{+}\right)^{2}} \partial_{c}\left(F_{U}(c(y, s), \tilde{c}(x, t))-F_{U}(\tilde{c}(x, t), c(y, s))\right) \mathbf{u}(x, t) \nabla_{y} c(y, s) \psi(t) \bar{w}(x-y) w(t-s) \mathrm{d} x \mathrm{~d} t \mathrm{~d} y \mathrm{~d} s \\
& \quad+\int_{\left(\mathbb{R}^{2} \times R^{+}\right)^{2}} \partial_{c} F_{U}(c(y, s), \tilde{c}(x, t))(\mathbf{u}(y, s)-\mathbf{u}(x, t)) \nabla_{y} c(y, s) \psi(t) \bar{w}(x-y) w(t-s) \mathrm{d} x \mathrm{~d} t \mathrm{~d} y \mathrm{~d} s
\end{aligned}
$$

Using Lemma 4.3 we get

$$
\begin{aligned}
I_{c} \leq & \|\psi\|_{L^{\infty}((0, T))}\|\mathbf{u}\|_{L^{\infty}\left(\mathbb{R}^{2} \times(0, T)\right)} \frac{k_{1}}{2} \delta\left\|f^{\prime \prime}\right\|_{L^{\infty}(\mathbb{R})} \int_{\mathbb{R}^{2} \times(0, T)}\left|\nabla_{y} c(y, s)\right| \mathrm{d} y \mathrm{~d} s \\
& +\left\|f^{\prime}\right\|_{L^{\infty}(\mathbb{R})} \int_{\left(\mathbb{R}^{2} \times R^{+}\right)^{2}}\left|\mathbf{u}(y, s)-\mathbf{u}(x, t) \| \nabla_{y} c(y, s)\right| \psi(t) \bar{w}(x-y) w(t-s) \mathrm{d} x \mathrm{~d} t \mathrm{~d} y \mathrm{~d} s \\
\leq & \|\psi\|_{L^{\infty}((0, T))}\left(\|\mathbf{u}\|_{L^{\infty}\left(\mathbb{R}^{2} \times(0, T)\right)} \frac{k_{1}}{2}\left\|f^{\prime \prime}\right\|_{L^{\infty}(\mathbb{R})} \int_{0}^{T}|c(t)|_{B V\left(\mathbb{R}^{2}\right)} \mathrm{d} t \delta\right. \\
& \left.+\left\|f^{\prime}\right\|_{L^{\infty}(\mathbb{R})}|\mathbf{u}|_{B V\left(\mathbb{R}^{2} \times(0, T)\right)} \int_{0}^{T}|c(t)|_{B V\left(\mathbb{R}^{2}\right)} \mathrm{d} t \frac{1}{r}\right),
\end{aligned}
$$

where $|\mathbf{u}|_{B V\left(\mathbb{R}^{2} \times(0, T)\right)}$ is defined in the Theorem.

The term $I_{d}$ : Writing the summands of the first integral separately and using integration by parts with respect to $y, x$ respectively, we get

$$
\begin{aligned}
I_{d}= & 2 \int_{\left(\mathbb{R}^{2} \times R^{+}\right)^{2}} D \nabla_{y} c(y, s) \cdot \nabla_{x} \tilde{c}(x, t) U^{\prime \prime}(\tilde{c}(x, t)-c(y, s)) \psi(t) \bar{w}(x-y) w(t-s) \mathrm{d} x \mathrm{~d} t \mathrm{~d} y \mathrm{~d} s \\
& -\int_{\left(\mathbb{R}^{2} \times R^{+}\right)^{2}} D U^{\prime \prime}(\tilde{c}(x, t)-c(y, s))\left(\left|\nabla_{x} \tilde{c}(x, t)\right|^{2}+\left|\nabla_{y} c(y, s)\right|^{2}\right) \psi(t) \bar{w}(x-y) w(t-s) \mathrm{d} x \mathrm{~d} t \mathrm{~d} y \mathrm{~d} s \\
= & -\int_{\left(\mathbb{R}^{2} \times R^{+}\right)^{2}} D U^{\prime \prime}(\tilde{c}(x, t)-c(y, s))\left|\nabla_{x} \tilde{c}(x, t)-\nabla_{y} c(y, s)\right|^{2} \psi(t) \bar{w}(x-y) w(t-s) \mathrm{d} x \mathrm{~d} t \mathrm{~d} y \mathrm{~d} s .
\end{aligned}
$$


As we can see, $I_{d}$ is negative. We therefore define $H(\tilde{c}-c):=-I_{d}$ and put the term to the left hand side.

\section{The term $I_{\lambda}$ :}

$$
\begin{aligned}
\left|I_{\lambda}\right| \leq & \int_{\left(\mathbb{R}^{2} \times R^{+}\right)^{2}}|\lambda(x, t) \| c(x, t)-\tilde{c}(x, t)| \psi(t) \bar{w}(x-y) w(t-s) \mathrm{d} x \mathrm{~d} t \mathrm{~d} y \mathrm{~d} s \\
& +\int_{\left(\mathbb{R}^{2} \times R^{+}\right)^{2}}|\lambda(y, s) c(y, s)-\lambda(x, t) c(x, t)| \psi(t) \bar{w}(x-y) w(t-s) \mathrm{d} x \mathrm{~d} t \mathrm{~d} y \mathrm{~d} s \\
& -\int_{\left(\mathbb{R}^{2} \times R^{+}\right)^{2}}|\lambda(x, t)||\tilde{c}(x, t)-c(x, t)| \psi(t) \bar{w}(x-y) w(t-s) \mathrm{d} x \mathrm{~d} t \mathrm{~d} y \mathrm{~d} s \\
= & \int_{\left(\mathbb{R}^{2} \times R^{+}\right)^{2}}|\lambda(y, s) c(y, s)-\lambda(x, t) c(x, t)| \psi(t) \bar{w}(x-y) w(t-s) \mathrm{d} x \mathrm{~d} t \mathrm{~d} y \mathrm{~d} s \\
\leq & \frac{1}{r}\|\psi\|_{L^{\infty}((0, T))}\left(\|c\|_{L^{\infty}\left(\mathbb{R}^{2} \times(0, T)\right)}|\lambda|_{B V\left(\mathbb{R}^{2} \times(0, T)\right)}+\|\lambda\|_{L^{\infty}\left(\mathbb{R}^{2} \times(0, T)\right)}|c|_{B V\left(\mathbb{R}^{2} \times(0, T)\right)}\right) .
\end{aligned}
$$

Summing up the inequalities above, we get the desired estimate.

With the approximation inequality (Th. 5.3) and the continuous entropy inequality for the discrete solution (Lem. 4.9 and Cor. 4.11) we have now all tools at hand to state and prove the main result of this paper.

Theorem 5.4 (A posteriori error estimate). Let $c$ be the entropy solution of (1) and $c_{h}$ the approximate solution, defined in 3.5. Furthermore, let the Assumptions 2.7, 3.1, 3.6 and the properties of the numerical fluxes (5)-(9), (17)-(21) be fulfilled. Then the following a posteriori error estimate holds for any $M>0$ such that $\|\lambda\|_{L^{\infty}\left(\mathbb{R}^{2} \times(0, T)\right)} \leq M$ :

$$
\left\|c_{h}-c\right\|_{L^{1}\left(\mathbb{R}^{2} \times(0, T)\right)}+\frac{1}{M} H\left(c_{h}-c\right) \leq \eta,
$$

where $\eta$ is given by

$$
\begin{aligned}
\eta:= & \frac{1}{M}\left(\eta_{0}+\eta_{p, 0}+\eta_{\lambda}+\left\|\psi_{t}\right\|_{L^{\infty}((0, T))}\left(\eta_{t}+\eta_{c}+\eta_{d}+\eta_{p, 1}\right)+2 \sqrt{k_{4} \eta_{U}}\right. \\
& \left.+2 \sqrt{k_{3}\|\psi\|_{L^{\infty}((0, T))}\left(K_{1}+K_{1}^{\prime}\right)\left(\eta_{t}+\eta_{c}+\eta_{d}+\eta_{p, 1}\right)}\right),
\end{aligned}
$$

where the constants $k_{3}$ and $k_{4}$ and $H\left(c_{h}-c\right)$ are defined in Theorem 5.3 and the error estimator terms $\eta_{i}, K_{1}$, $K_{1}^{\prime}$ are defined in Lemma 4.11. Note that $H\left(c_{h}-c\right)$ is positive for all values $c_{h}$ and $c$.

Proof. From Theorem 5.3 and Corollary 4.11 we have

$$
\| c_{h}-\left.c\right|_{L^{1}\left(\mathbb{R}^{2} \times(0, T)\right)}+\frac{1}{M} H\left(c_{h}-c\right) \leq \frac{1}{M}\left(k_{3} \frac{1}{r}+k_{4} \delta+\bar{R}_{h}\left(\eta_{0}, \eta_{t}, \eta_{c}, \eta_{d}, \eta_{\lambda}, \eta_{p, 0}, \eta_{p, 1}, \eta_{U}\right)\right),
$$

where $\bar{R}_{h}$ is defined in Lemma 4.11. Minimizing the right hand side with respect to the parameters $r$ and $\delta$ yields the desired result.

Remark 5.5. In the situation where $\lambda \equiv 0, M$ can be chosen as $\ln 2 / T$. Thus, $\|\psi\|_{L^{\infty}((0, T))}=1$ and the right hand side of the a posteriori error estimate does not depend exponentially on $T$.

In this section we have proved an a posteriori error estimate for an implicit vertex centered finite volume approximation ( $c f$. Def. 3.5) of the scalar nonlinear convection-diffusion-reaction equation (1).

So far, the a posteriori error estimate is an upper bound for the error but we were not able to prove also efficiency of the estimator, which corresponds to a lower bound of the error by the estimator. We also could 
not prove a convergence rate for the error estimator until now, which would then give us also an a priori error estimate. In order to justify the obtained error estimator at least numerically, we are now going to design a mesh-adaptive solution strategy for the numerical scheme.

\section{Adaptive strategy And numerical EXPERIMEnts}

In this section we confirm the theoretical results of the last section by some numerical tests in two space dimensions. Therefore, we first introduce an adaptive algorithm which is based on the a posteriori error estimate of Theorem 5.4. In [41] we have already given an adaptive refinement strategy in the case of a cell centered explicit finite volume scheme. Thereby we followed mostly the ideas of [33], where an adaptation strategy was also given for an explicit finite volume scheme, but for hyperbolic conservation laws.

In contrast to the cited works, we have to introduce a completely different adaptation strategy in this situation because we deal here with an implicit finite volume scheme, where no correlation between the space discretization parameter $h$ and the time step $\Delta t$ has to be fulfilled. Therefore, we have to deduce an adaptation strategy from the error estimator which dynamically adapts the time step $\Delta t$ independently of the local grid size $h$. A second consequence of the implicit scheme is that information may be transported with "infinite speed" through the domain, whereas an explicit scheme transports information at most from one grid cell to the neighbouring cells in one time step. This is guaranteed by the CFL-condition. Thus, the adaptive algorithm for the implicit scheme must be capable to follow steep fronts not only from one cell to the next, but through the whole domain. Finally, the implicit scheme is defined on the "dual cells" $\Omega_{j}$ and not on the triangular mesh $\mathcal{T}$. As we are not able to coarse and refine the dual cells directly, we have to define local adaptation criteria on the underlying triangular mesh and to adapt the triangulation. The adapted dual mesh is then automatically defined as the dual mesh of the adapted triangulation. Thus, two additional problems have to be solved. First we have to define local error indicators on the primal triangles of the mesh and second we have to define conservative prolongation and restriction operators for the solution between to successive grids.

In the next subsection we will first define local error indicators on the triangles which are motivated by the a posteriori error estimate of Theorem 5.4. Of course our computations can only be done in a bounded domain $\Omega \subset \mathbb{R}^{2}$. Therefore, let us denote the triangulation of $\Omega$ at time $t^{n}$ by $\mathcal{T}^{n}$, the set of triangle indices of $\mathcal{T}^{n}$ by $K^{n}$ and the set of vertex indices of $\mathcal{T}^{n}$ by $I^{n}$.

\subsection{Local error indicators}

For the definition of an adaptive algorithm let us for simplicity look at the situation, where the velocity field $\mathbf{u} \equiv \mathbf{v}=$ const. Due to the contribution to the estimator in Theorem 5.4 let us define for $k \in K^{n}, t^{n} \in J$ the following local estimator quantities

$$
\begin{aligned}
&\left(\eta_{0}\right)_{k}:= \int_{T_{k}}\left|c_{0}(x)-c_{h}^{0}(x)\right| \mathrm{d} x, \\
&\left(\eta_{t}\right)^{n}:= \sum_{j \in I^{n}}\left|c_{j}^{n+1}-c_{j}^{n}\right| \Delta t^{n}\left|\Omega_{j}\right|, \\
&\left(\eta_{c}\right)_{k}^{n}:=\sum_{\left\{(j, l, *) \in \mathcal{E}^{*} \mid S_{j l}^{*} \cap T_{k} \neq \emptyset\right\}}\left(h_{j l}+\Delta t^{n}\right) \Delta t^{n} Q_{j l}^{*, n+1}\left(c_{j}^{n+1}, c_{l}^{n+1}\right)\left|c_{j}^{n+1}-c_{l}^{n+1}\right|, \\
&\left(\eta_{d}\right)_{k}^{n}:=\frac{1}{2} \sum_{\left\{(j, l) \in \mathcal{E} \mid \Gamma_{j l} \cap T_{k} \neq \emptyset\right\}} D\left[\nabla c_{h}^{n+1} \cdot \mathbf{m}_{j l}\right]_{\Gamma_{j l}} \Delta t^{n}\left|\Gamma_{j l}\right|\left(h_{j l}^{n}+\Delta t^{n}\right), \\
&\left(\eta_{\lambda}\right)_{k}^{n}:=\sum_{\left\{j \in I^{n} \mid \Omega_{j} \cap T_{k} \neq \emptyset\right\}}\left|c_{j}^{n+1}\right| \int_{t^{n}}^{t^{n+1}} \int_{\Omega_{j} \cap T_{k}}\left|\lambda_{j}^{n+1}-\lambda(x, t)\right| \mathrm{d} x \mathrm{~d} t, \\
&\left(\eta_{h}\right)_{k}^{n}:=\left(\eta_{c}\right)_{k}^{n}+\left(\eta_{d}\right)_{k}^{n}+\left(\left(\eta_{\lambda}\right)_{k}^{n}\right)^{2} .
\end{aligned}
$$


Using these definition let us define the global in space estimators

$$
\eta_{0}:=\sqrt{\sum_{k \in K^{0}}\left(\left(\eta_{0}\right)_{k}\right)^{2}}, \quad\left(\eta_{h}\right)^{n}:=\sum_{k \in K^{n}}\left(\eta_{h}\right)_{k}^{n}
$$

and finally the global in time and space estimator

$$
\eta_{t}:=\sum_{t^{n} \in J}\left(\eta_{t}\right)^{n}, \quad \eta_{h}:=\sum_{t^{n} \in J}\left(\eta_{h}\right)^{n}, \quad \eta:=\sqrt{K_{0}\left(\eta_{0}\right)^{2}+K_{t} \eta_{t}+K_{h} \eta_{h}}
$$

Here $K_{0}, K_{t}$ and $K_{h}$ have to be chosen corresponding to the estimate in Theorem 5.4 and $\eta$ corresponds to the error estimator in the Theorem. Of course this correspondence is not exactly fulfilled, as we do not take care of the higher order terms in the estimate and neglect the terms $\eta_{p, 0}, \eta_{p, 1}$ and $\eta_{U}$.

Before we define the adaptive algorithm let us introduce a prescribed global tolerance TOL and some local tolerances which split up the global one. Therefore, let the factors $\Theta_{0}, \Theta_{t}, \Theta_{h}>0$ be given such that $\Theta_{0}+\Theta_{t}+$ $\Theta_{h}=1$. Now we define

$$
\begin{aligned}
\mathrm{TOL}_{0} & :=\Theta_{0} \mathrm{TOL}^{2}, & \left(\mathrm{TOL}_{0}\right)_{k}:=\mathrm{TOL}_{0} / \bar{M}^{n} \\
\left(\mathrm{TOL}_{h}\right)^{n} & :=\frac{\Delta t^{n}}{T} \Theta_{h} \mathrm{TOL}^{2}, & \left(\mathrm{TOL}_{h}\right)_{k}^{n}:=\left(\mathrm{TOL}_{h}\right)^{n} / \bar{M}^{n} \\
\left(\mathrm{TOL}_{t}\right)^{n} & :=\frac{\Delta t^{n}}{T} \Theta_{t} \mathrm{TOL}^{2}, &
\end{aligned}
$$

where $\bar{M}^{n}$ denotes the number of triangles in $\mathcal{T}^{n}$. With this definition we obviously have

$$
\mathrm{TOL}_{0}=\sum_{k \in K^{0}}\left(\mathrm{TOL}_{0}\right)_{k}, \quad\left(\mathrm{TOL}_{h}\right)^{n}=\sum_{k \in K^{n}}\left(\mathrm{TOL}_{h}\right)_{k}^{n}, \text { and } \quad \mathrm{TOL}=\sqrt{\mathrm{TOL}_{0}+\sum_{t^{n} \in J}\left(\mathrm{TOL}_{t}\right)^{n}+\sum_{t^{n} \in J}\left(\mathrm{TOL}_{h}\right)^{n}} .
$$

\subsection{Prolongation and restriction operators}

With the definition of the error indicators and tolerances of the last subsection we have nearly everything at hand to define the adaptive solution algorithm. The only thing missing is the non trivial conservative prolongation and restriction of the approximate solution between two meshes. Therefore, in this section we will define the refinement and coarsening of the underlying triangular mesh and, based on this, we will define the prolongation and restriction operators.

As a grid refinement and coarsening procedure we choose a recursive bisection algorithm, where a triangle of the mesh is refined by introducing a new vertex on a marked edge of the triangle. In oder to get a conform triangulation the algorithm ensures, that the corresponding neighbouring cell will be refined as well. It can be shown that under certain assumptions on the macro triangulation, this recursion is finite and that the refinement remains local. For a detailed definition of the refinement procedure we refer to [5]. We stress that the refinement procedure ensures shape regularity of the successively refined meshes. In Figure 2 the grid refinement is demonstrated. The coarsening procedure is likely the inversion of the refinement algorithm. Therefore, the algorithm searches for vertices in the triangulation which can be removed in such a way that the bisection of two neighbouring cells is inverted. Figure 3 demonstrates this situation. Having this refinement and coarsening procedures in mind we are now going to define the prolongation and restriction operators between two successively refined or coarsened grids.

\subsubsection{Prolongation}

Let us look at the local situation, where the triangles $\tilde{T}_{j l}^{a}$ and $\tilde{T}_{j l}^{b}$ are refined by inserting a new vertex $p_{i}:=p_{j l}$ in the midpoint of the edge $\Gamma_{j l}$. This situation is sketched in Figure 4. By this local refinement procedure the 


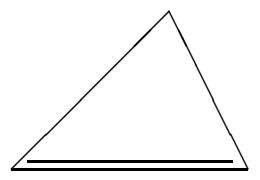

Refinement

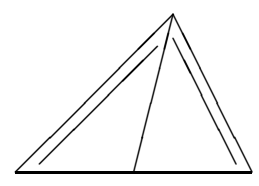

FIGURE 2. Refinement of a triangle by bisection. The marked edges are to be refined in the next refinement step.

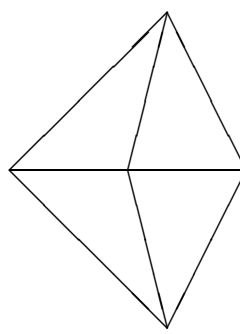

\section{Coarsening}

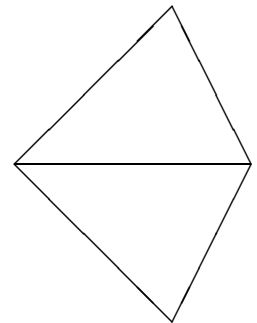

Figure 3. Coarsening of triangles by inverse bisection.

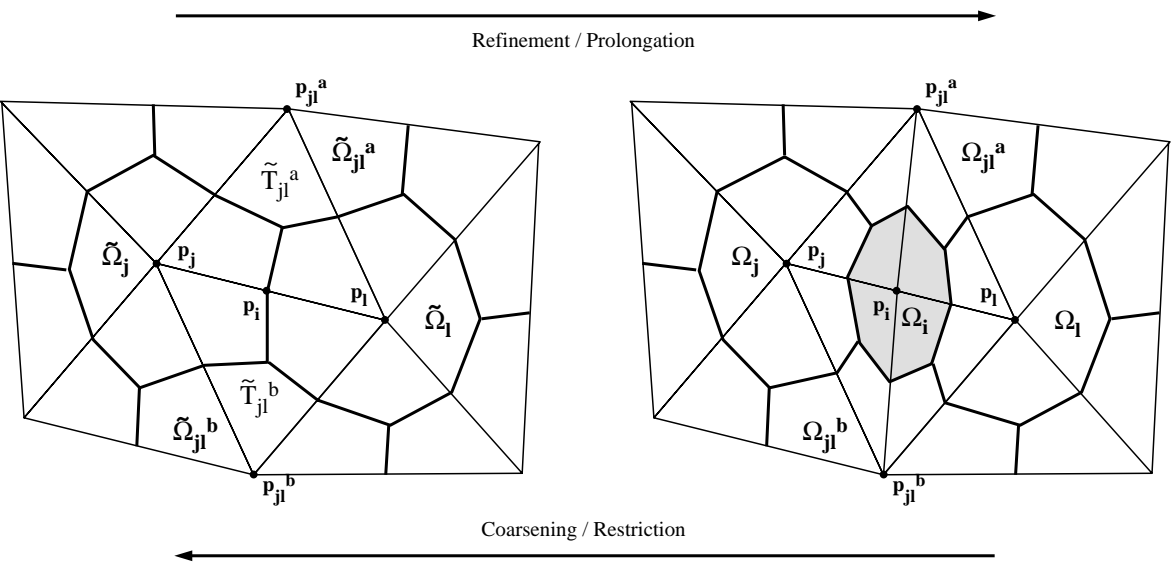

FIGURE 4. Sketch of the primal triangular grid and the corresponding dual cells in a refinement or coarsening situation.

dual cells $\Omega_{j}, \Omega_{l}$ and $\Omega_{j l}^{a}, \Omega_{j l}^{b}$ are changed and a new dual cell $\Omega_{i}$ is inserted. To differ between the old and new cells let us denote the old ones by $\tilde{\Omega}_{j}, \tilde{\Omega}_{l}$ and $\tilde{\Omega}_{j l}^{a}, \tilde{\Omega}_{j l}^{b}$. In the same manner we denote the old values of the approximate solution by $\tilde{c}_{j}, \tilde{c}_{l}, \tilde{c}_{j l}^{a}$ and $\tilde{c}_{j l}^{b}$.

Corresponding to the definition of the dual cells we have the following relations between the volumes of those dual cells, where $* \in \mathcal{P} M$ :

$$
\begin{aligned}
\left|\tilde{\Omega}_{j} \cap \tilde{T}_{j l}^{*}\right|=\left|\tilde{\Omega}_{l} \cap \tilde{T}_{j l}^{*}\right| & =\frac{1}{3}\left|\tilde{T}_{j l}^{*}\right|, \quad\left|\Omega_{j} \cap T_{j i}^{*}\right|=\frac{1}{3}\left|T_{j i}^{*}\right|=\frac{1}{2}\left|\tilde{\Omega}_{j} \cap \tilde{T}_{j l}^{*}\right|, \quad\left|\Omega_{l} \cap T_{j i}^{*}\right|=\frac{1}{3}\left|T_{j i}^{*}\right|=\frac{1}{2}\left|\tilde{\Omega}_{l} \cap \tilde{T}_{j l}^{*}\right|, \\
\left|T_{j i}^{*}\right| & =\frac{1}{2}\left|\tilde{T}_{j l}^{*}\right|, \quad\left|\Omega_{i} \cap T_{j i}^{*}\right|=\left|\Omega_{j} \cap T_{j i}^{*}\right|, \quad\left|\tilde{\Omega}_{j l}^{*}\right|=\left|\Omega_{j l}^{*}\right| .
\end{aligned}
$$


This relations induce that for a conservative prolongation we do not need to change the values $\tilde{c}_{j}, \tilde{c}_{l}, \tilde{c}_{j l}^{a}, \tilde{c}_{j l}^{b}$ and the new value $c_{i}$ should be defined as

$$
c_{i}:=\frac{1}{2}\left(\tilde{c}_{j}+\tilde{c}_{l}\right)
$$

We therefore define the local prolongation operator $\mathbf{P R O L}_{j l}$ as

$$
\mathbf{P R O L}_{j l}(u, v, r, s):=(u, v, r, s, 0.5(u+v)),
$$

such that $\left(c_{j}, c_{l}, c_{j l}^{a}, c_{j l}^{b}, c_{i}\right):=\mathbf{P R O L}_{j l}\left(\tilde{c}_{j}, \tilde{c}_{l}, \tilde{c}_{j l}^{a}, \tilde{c}_{j l}^{b}\right)$. We point out that with this definition of the prolongation operator the following properties are fulfilled:

1) $\tilde{c}_{h}=c_{h}$ if $c_{h}$ is the prolongation of $\tilde{c}_{h}$,

2) $\left|\tilde{\Omega}_{j}\right| \tilde{c}_{j}+\left|\tilde{\Omega}_{j}\right| \tilde{c}_{l}=\left|\Omega_{j}\right| c_{j}+\left|\Omega_{l}\right| c_{l}+\left|\Omega_{i}\right| c_{i}$.

Especially the prolongation conserves the mass locally. The global prolongation operator PROL is defined by applying the local prolongation operators to all patches (pairs of triangles) which are refined.

\subsubsection{Restriction}

In the situation of local coarsening we are exactly in the opposite situation as described in the last subsection. Here the vertex $p_{i}$ is to be removed and we have to define a local restriction operator $\operatorname{REST}_{j l}$ which distributes the value $c_{i}$ to the neighbouring values $c_{j}$ and $c_{l}$. As in the prolongation step the values $c_{j l}^{*}$ do not need to be changed, as the volume of the corresponding cells do not change their values by the coarsening procedure $\left(\left|\tilde{\Omega}_{j l}^{*}\right|=\left|\Omega_{j l}^{*}\right|\right)$. In order to define a local conservative restriction operator let us first define

$$
V_{j i}:=\left|\Omega_{j} \cap T_{j i}^{a}\right|+\left|\Omega_{j} \cap T_{j i}^{b}\right|
$$

and $V_{l i}$ respectively. A sufficient condition for local conservation is then given by the relations

$$
\left|\Omega_{j}\right| c_{j}+V_{j i} c_{i}=\left|\tilde{\Omega}_{j}\right| \tilde{c}_{j}, \quad\left|\Omega_{j}\right| c_{l}+V_{l i} c_{i}=\left|\tilde{\Omega}_{l}\right| \tilde{c}_{l},
$$

where we mark the new values by tilde. Taking into account that $\left|\tilde{\Omega}_{j}\right|=\left|\Omega_{j}\right|+V_{j i}$ and $\left|\tilde{\Omega}_{l}\right|=\left|\Omega_{l}\right|+V_{l i}$ this leads to the following definition of the new values $\tilde{c}_{j}, \tilde{c}_{l}$.

$$
\tilde{c}_{j}:=\frac{\left|\Omega_{j}\right| c_{j}+V_{j i} c_{i}}{\left|\Omega_{j}\right|+V_{j i}}, \quad \tilde{c}_{l}:=\frac{\left|\Omega_{l}\right| c_{l}+V_{l i} c_{i}}{\left|\Omega_{l}\right|+V_{l i}}
$$

Accordingly we define the local restriction operator by

$$
\boldsymbol{R E S T}_{j l}(u, v, r, s, w):=\left(\frac{\left|\Omega_{j}\right| u+V_{j i} w}{\left|\Omega_{j}\right|+V_{j i}}, \frac{\left|\Omega_{l}\right| v+V_{l i} w}{\left|\Omega_{l}\right|+V_{l i}}, r, s\right)
$$

such that the new values are given as $\left(\tilde{c}_{j}, \tilde{c}_{l}, \tilde{c}_{j l}^{a}, \tilde{c}_{j l}^{b}\right):=\mathbf{R E S T}_{j l}\left(c_{j}, c_{l}, c_{j l}^{a}, c_{j l}^{b}, c_{i}\right)$.

As in the case of the prolongation, we remark that this definition of the restriction operator ensures local mass conservation, but in contrast to a standard restriction the values at the coarse grid nodes are changed. Again, we define the global restriction operator REST by applying the local ones to patches which are to be coarsened. 


\subsection{Adaptive solution algorithm}

Let the error indicator and the tolerances be defined as in Subsection 6.1 and the prolongation and restriction operators as in Subsection 6.2. Then we define the solution algorithm as follows:

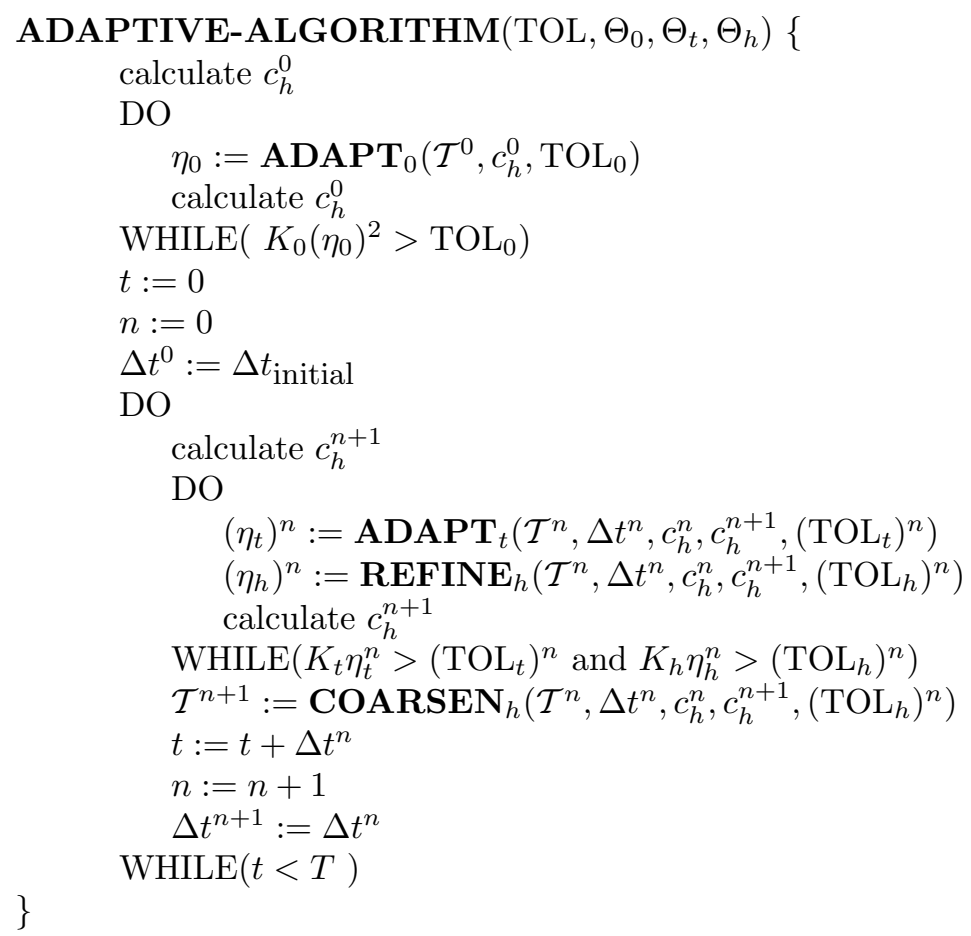

Here the subroutines $\mathbf{A D A P T}_{0}, \mathbf{R E F I N E}_{h}$, and $\mathbf{C O A R S E N}{ }_{h}$ and $\mathbf{A D A P T}$ are given as follows.

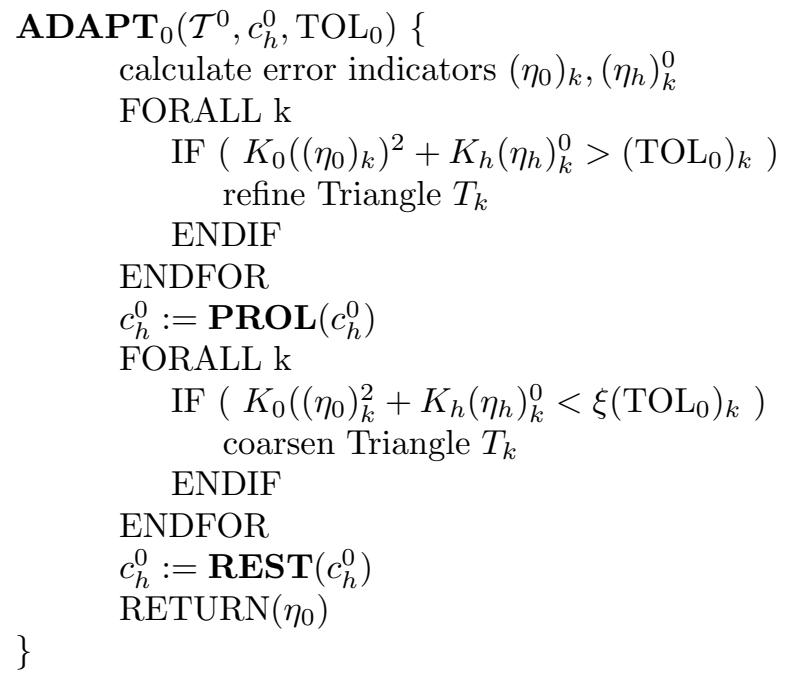

In the definition of this subroutine and in the sequel $\xi \in(0,1)$ denotes a fixed constant which should be chosen in dependence of the refinement and coarsening procedure. For the bisection algorithm we usually choose $\xi=0.5$. We stress that the subroutine returns the calculated estimator value $\eta_{0}$, the updated triangulation $\mathcal{T}^{0}$ and the updated solution vector $c_{h}^{0}$. 

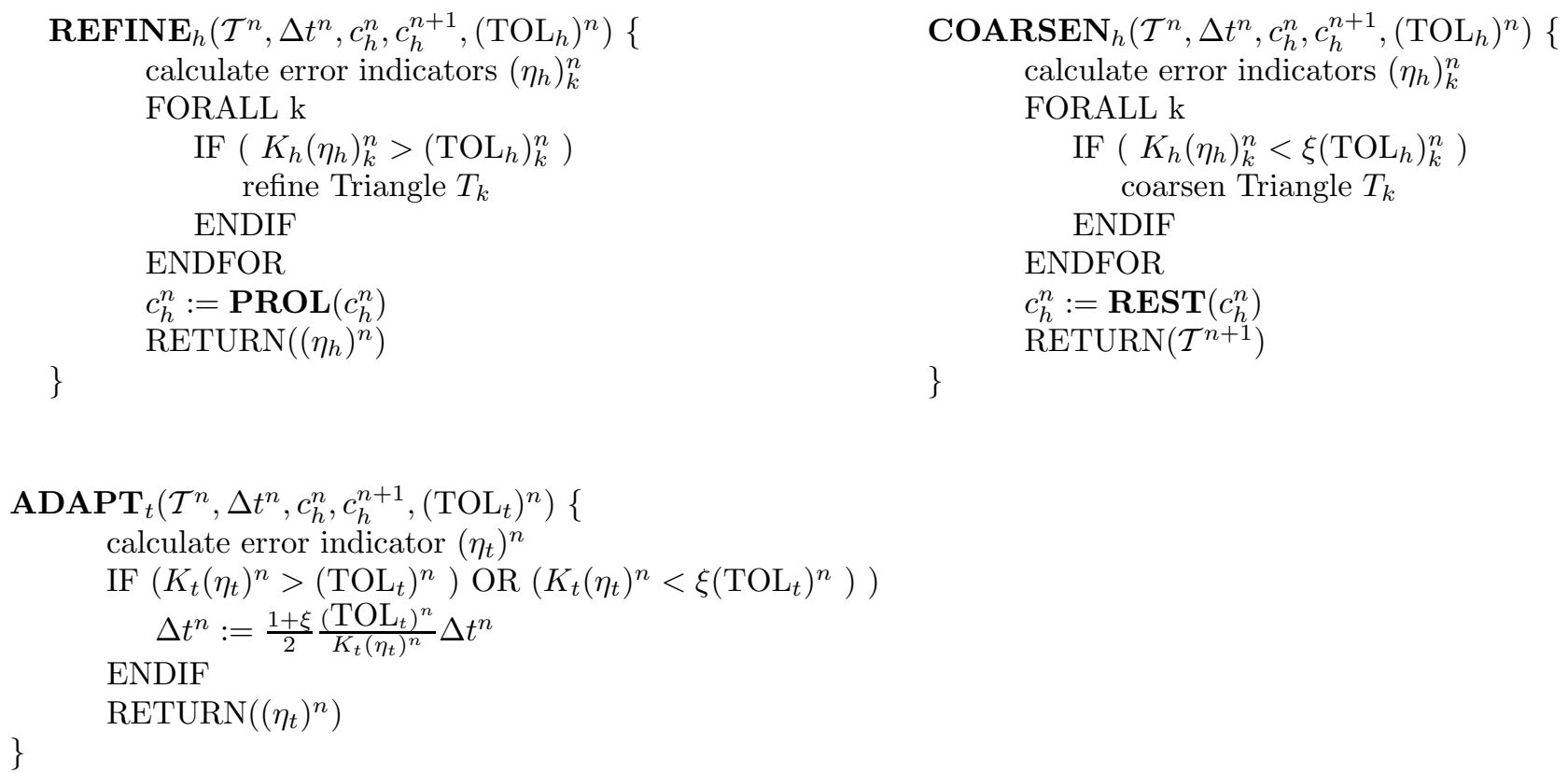

Let us remark that the definition of the new time step in this subroutine is chosen under the assumption that $\left(\eta_{t}^{n}\right)=\mathcal{O}\left(\left(\Delta t^{n}\right)^{2}\right)$. As the tolerance $\left(\mathrm{TOL}_{h}\right)_{h}^{n}$ is also influenced by the variation of $\Delta t^{n}$, the definition in the subroutine guaranties that the new time step is chosen such that the new error indicator is smaller than the new tolerance. We further mention that the relation between the error indicator in space $\left(\left(\eta_{h}\right)^{n}\right)$ and the tolerance in space $\left(\left(\mathrm{TOL}_{h}\right)^{n}\right)$ is independent of $\Delta t^{n}$ and the refinement procedure is therefore not influenced by the variation of $\left(\mathrm{TOL}_{h}\right)_{n}$ with $\Delta t^{n}$. With this adaptive strategy we expect from the a posteriori error estimate of Theorem 5.4 that we get approximately $\left\|c-c_{h}\right\|_{L^{1}} \leq \eta \leq$ TOL. In the following numerical experiment we will show this relation numerically.

\subsection{Numerical experiments}

In this subsection we apply the adaptive algorithm defined in Subsection 6.3 to some test problems in order to demonstrate the applicability and efficiency of the adaptive solution scheme. As our aim is a comparison with the results for the explicit cell centered finite volume scheme, we choose the same examples as in [41]. Note that the main difference between the error estimator and the adaptation strategy for the explicit cell centered scheme in [41] and the implicit vertex centered scheme in this paper is the treatment of the error coming from the diffusive part and the fully adaptive time discretization for the implicit method.

\subsubsection{First example:}

As a first example we choose a linear problem, where the exact solution is known. Thus we can compare the $L^{1}$-error between the exact and the approximate solution with the error estimator $\eta$, where $\eta$ is defined in Subsection 6.1.

We look at the following Cauchy problem in $\mathbb{R}^{2}$ :

$$
\begin{aligned}
c_{t}+\nabla \cdot((1,0) c)-\varepsilon \Delta c & =0 \text { in } \mathbb{R}^{2} \times(0, T), \\
c(\cdot, 0) & =\frac{1}{2} \operatorname{sign}\left(x_{1}\right)+\frac{1}{2} \text { in } \mathbb{R}^{2} .
\end{aligned}
$$

Then $c(x, t)=\operatorname{erf}\left(\left(x_{1}-t\right) / \sqrt{4 \varepsilon t}\right)$, where $\left.\operatorname{erf}(s):=1 / \sqrt{(} \pi\right) \int_{-\infty}^{s} \mathrm{e}^{-r^{2}} \mathrm{~d} r$ denotes the error function, is the exact solution of this problem. For the numerical computation we look at this Riemann problem on the bounded 


\begin{tabular}{|c||c|c|c|c|}
\hline$h_{\min }$ & $\left\|c-c_{h}\right\|_{L^{1}}$ & $\mathrm{EOC}\left(\left\|c-c_{h}\right\|_{L^{1}}\right)$ & estimator $\eta$ & $\mathrm{EOC}(\eta)$ \\
\hline \hline $3.536 \mathrm{E}-01$ & $4.26051 \mathrm{E}-01$ & & $4.34711 \mathrm{E}-01$ & \\
$1.768 \mathrm{E}-01$ & $2.72064 \mathrm{E}-01$ & 0.64710 & $3.07580 \mathrm{E}-01$ & 0.49910 \\
$8.839 \mathrm{E}-02$ & $1.80522 \mathrm{E}-01$ & 0.59175 & $2.16751 \mathrm{E}-01$ & 0.50492 \\
$4.419 \mathrm{E}-02$ & $1.26491 \mathrm{E}-01$ & 0.51314 & $1.54180 \mathrm{E}-01$ & 0.49142 \\
$2.210 \mathrm{E}-02$ & $8.85093 \mathrm{E}-02$ & 0.51514 & $1.09156 \mathrm{E}-01$ & 0.49822 \\
$1.105 \mathrm{E}-02$ & $6.20374 \mathrm{E}-02$ & 0.51269 & $7.71871 \mathrm{E}-02$ & 0.49996 \\
\hline
\end{tabular}

TABLE 1. Comparison between the error estimator and the actual error for the linear problem (24) with $\varepsilon=1 E-06$ and $T=1$ on uniform grids. EOC denotes the experimental order of convergence. As we are free in the choice of the time step for the implicit scheme we chose $\mathrm{CFL}=15$ in this computation. Let us remark that we should expect a better absolute error for smaller CFL numbers, but that the convergence history should stay the same.

\begin{tabular}{|c||c|c|c|c|c|l|}
\hline TOL & $\left\|c-c_{h}\right\|_{L^{1}}$ & $\frac{\text { TOL }}{\left\|c-c_{h}\right\|_{L^{1}}}$ & estimator $\eta$ & $\frac{\text { TOL }}{\eta}$ & $\Delta t$ & $\bar{M}^{N}$ \\
\hline \hline 1.1314 & $2.7651 \mathrm{E}-01$ & 4.101 & $9.6503 \mathrm{E}-01$ & 1.172 & $9.60 \mathrm{E}-02$ & 105 \\
0.8000 & $1.9282 \mathrm{E}-01$ & 4.149 & $7.0893 \mathrm{E}-01$ & 1.128 & $4.80 \mathrm{E}-02$ & 296 \\
0.5657 & $1.3498 \mathrm{E}-01$ & 4.190 & $5.1182 \mathrm{E}-01$ & 1.105 & $2.40 \mathrm{E}-02$ & 966 \\
0.4000 & $9.4807 \mathrm{E}-02$ & 4.219 & $3.6763 \mathrm{E}-01$ & 1.088 & $1.20 \mathrm{E}-02$ & 2896 \\
0.2828 & $6.6136 \mathrm{E}-02$ & 4.276 & $2.6256 \mathrm{E}-01$ & 1.077 & $5.72 \mathrm{E}-03$ & 9387 \\
0.2000 & $4.7063 \mathrm{E}-02$ & 4.255 & $1.8754 \mathrm{E}-01$ & 1.066 & $3.00 \mathrm{E}-03$ & 26085 \\
\hline
\end{tabular}

TABLE 2. Comparison between the prescribed tolerance, the actual error and the error estimator for the linear problem $(24)$ with $\varepsilon=1 E-06$ using the adaptive solution algorithm $(T=1.0)$. The last two columns show average time step and the number of triangles in the underlying triangulation at the last time step.

domain $\Omega=(-0.5,1.5) \times(0,1)$, were we prescribe periodic boundary conditions on the upper and lower boundary and Dirichlet boundary conditions (exact solution) on the left and right boundary of the domain.

Table 1 shows a comparison of the $L^{1}$-error between the exact and the approximate solution with the error estimator $\eta$ on uniform meshes. We recall that $\eta$ corresponds to the lowest order term in the a posteriori error estimate of Theorem 5.4. In order to compare the convergence rate between the error estimator and the actual error let us define for any nonnegative quantity $q_{h}$ depending on the uniform grid size $h$ the experimental order of convergence (EOC) as

$$
\operatorname{EOC}\left(q_{h}\right)=\ln \left(q_{2 h} / q_{h}\right) / \ln 2 .
$$

From Table 1 it can be seen that the error estimator converges with the same rate, as the error itself. This demonstrates that the global error estimator is also efficient.

In the next step we analyze the efficiency of the adaptive algorithm. Therefore we compute the solution of problem (24) with our adaptive algorithm for different prescribed tolerances. In Table 2 the square root of this prescribed global tolerance is given and a comparison with the actual $L^{1}$-error of the approximate solution and the square root of the estimator $\eta$ is shown. In order to compare the prescribed tolerance with the error and the estimator values we also give the corresponding ratios. We see that the ratio between the tolerance and $\eta$ converges to one which would be optimal. This indicates that our adaptive strategy and the definition of local 
error indicators is efficient. Furthermore, the ratio between the tolerance and the actual $L^{1}$-error nearly remains constant which again shows the efficiency of the estimator. The last two columns of Table 2 show the time step $\Delta t$ which remains constant in this experiment, and the number of triangles in the underlying triangulation at the last time step. It can be seen that the time step is bisected from one calculation to the next and that the number of triangles is approximately tripled from one calculation to the next. In a uniform calculation $(\Delta t$ and $h$ fixed) we expect the error to be of order $\sqrt{h+\Delta t}$ which was also the result of Table 1 . This means, for a bisection of the error we have to take $h / 4$ and $\Delta t / 4$ as parameters for the triangulation and the partition of the time interval which leads to a factor of 16 between the number of triangles in the primal and the refined grid. In the case of our adaptive algorithm this factor is reduced to 9 , whereas the time step is also divided by four. Thus, we can say that not only the error estimator is efficient, but also the adaptive strategy.

\subsubsection{Second example:}

As a second example we look at the following scalar nonlinear and degenerate parabolic equation for the saturation $s$ on $\Omega=(0,1) \times(0,0.5) \subset \mathbb{R}^{2}$ which models immiscible two phase flow in porous media

$$
\Phi s_{t}+\nabla \cdot(\mathbf{u} f(s))-\nabla \cdot(K D(s) \nabla s)=0,
$$

where $\mathbf{u}=(1,0), f(s)$ is the fractional flow rate and $D(s)$ the capillary diffusion given by $f(s)=\lambda_{1}(s) /\left(\lambda_{1}(s)+\right.$ $\left.\lambda_{2}(s)\right), D(s)=\lambda_{2}(s) f(s) p_{c}^{\prime}(s)$. The mobilities $\lambda_{1}, \lambda_{2}$ of the phases and the capillary pressure $p_{c}$ are given by $\lambda_{1}(s)=s^{3} /\left(2 \mu_{1}\right), \lambda_{2}(s)=(1-s)^{3} / \mu_{2}$ and $p_{c}(s)=-\sqrt{(1-s) / s}$. Furthermore, in this example we choose the viscosities $\mu_{1}=1, \mu_{2}=3$, the porosity $\Phi=0.2$ and the permeability $K=0.2$. As initial condition we choose $s \equiv 0$ in $(0.1,1) \times(0,0.5)$ and $s \equiv 1$ in $(0.1,1) \times(0,0.5)$ and as boundary conditions we impose $s=1$ on $\{0\} \times(0,0.5)$ and homogeneous Neumann boundary conditions on all other boundaries. Let us remark that this is the same problem as we dealt with in [41], where the initial conditions were shifted by 0.1 . Thus, we omit the resolution of the Dirichlet boundary conditions here. This "Buckley-Leverett" type problem is highly nonlinear in both the convection and diffusion term. Additionally the diffusion term which depends on the solution $s$ is degenerate for $s=0$ and $s=1$. As a solution we expect in the hyperbolic case $(D \equiv 0)$ a rarefaction wave followed by a shock. In the degenerate parabolic case which we examine here we have the same coarse structure of the solution, but the shock is smeared out in the upper part by the diffusion term which models capillary pressure effects. We refer to [11] for a more detailed discussion of the problem.

Figure 5 shows the result of the adaptive numerical scheme. The adaptively refined grid shows that the error estimator detects the regions of steep gradients very well. The profile of the saturation distribution computed with the adaptive algorithm on a horizontal slice is shown in Figure 6. Comparing these results with those obtained in [41], we see that the refinement of the mesh is concentrated more in the region of the steep gradient here. This is due to the fact, that the error indicator related to the diffusive part has the wrong order of convergence in [41] and overestimates the error outside the steep gradient. Therefore, especially the smooth regions of the solution are treated more appropriate here.

\section{Conclusion}

In this paper, we established robust a posteriori error estimates for finite volume approximations of convection dominated transport equations. The key to uniform error estimates for arbitrary large diffusion coefficients was the exploitation of the dissipation term in the entropy inequality. As these techniques require the dissipation term to be also well defined for the approximate solution, we dealt with vertex centered finite volume schemes in the analysis. Thus, for the vertex centered finite volume approximation, we obtained uniform a posteriori error estimates which on the one hand recover the results obtained in [33] for the hyperbolic case $(D \equiv 0)$, and on the other hand provide an robust extension of those estimates for arbitrary large diffusion coefficients $(D>0)$. Finally, we derived a numerical solution algorithm for the implicit vertex centered scheme which is adaptive both in the time and space discretization. Numerical experiments demonstrated the applicability and efficiency of the adaptive method. 

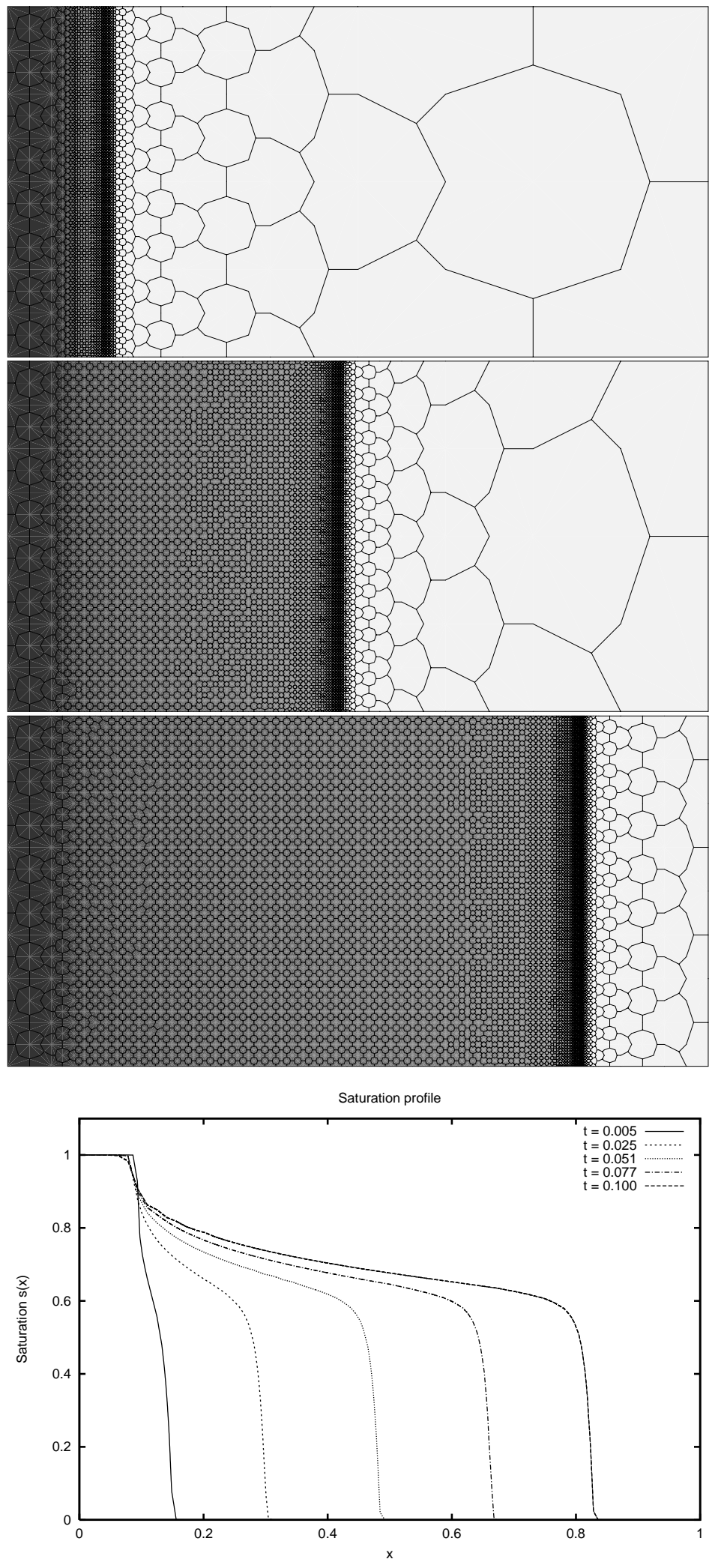

FIGURE 5. Adaptive dual mesh and saturation distribution for the degenerate parabolic problem at $t=0.005, t=0.051$ and $t=0.1$.

Figure 6. Profile of the saturation distribution of the degenerate parabolic problem on a horizontal slice at $t=0.005$, $t=0.025, t=0.051, t=0.077$ and $t=0.1$. 
Acknowledgements. The author would like to thank the referees for some precise suggestions that improved the presentation of this paper.

\section{REFERENCES}

[1] L. Angermann, An introduction to finite volume methods for linear elliptic equations of second order. Preprint 164, Institut für Angewandte Mathematik, Universität Erlangen (1995).

[2] Lutz Angermann, A finite element method for the numerical solution of convection-dominated anisotropic diffusion equations. Numer. Math. 85 (2000) 175-195.

[3] P. Angot, V. Dolejší, M. Feistauer and J. Felcman, Analysis of a combined barycentric finite volume - finite element method for nonlinear convection diffusion problems. Appl. Math., Praha 43 (1998) 263-311.

[4] I. Babuška and W.C. Rheinboldt, Error estimators for adaptive finite element computations. SIAM J. Numer. Anal. 15 (1978) $736-754$.

[5] E. Bänsch, Local mesh refinement in 2 and 3 dimensions. IMPACT Comput. Sci. Engrg. 3 (1991) 181-191.

[6] R. Becker and R. Rannacher, A feed-back approach to error control in finite element methods: Basic analysis and examples. East-West J. Numer. Math. 4 (1996) 237-264.

[7] F. Bouchut and B. Perthame, Kruzkov's estimates for scalar conservation laws revisited. Trans. Amer. Math. Soc. 350 (1998) $2847-2870$.

[8] J. Carrillo, Entropy solutions for nonlinear degenerate problems. Arch. Ration. Mech. Anal. 147 (1999) $269-361$.

[9] C. Chainais-Hillairet, Finite volume schemes for a nonlinear hyperbolic equation. Convergence towards the entropy solution and error estimates. ESAIM: M2AN 33 (1999) 129-156.

[10] S. Champier, Error estimates for the approximate solution of a nonlinear hyperbolic equation with source term given by finite volume scheme. Preprint, UMR 5585, Saint-Étienne University (1998).

[11] G. Chavent and J. Jaffre, Mathematical models and finite elements for reservoir simulation. Elsevier, New York (1986).

[12] B. Cockburn, F. Coquel and P.G. Lefloch, An error estimate for finite volume methods for multidimensional conservation laws. Math. Comput. 63 (1994) 77-103.

[13] B. Cockburn and H. Gau, A posteriori error estimates for general numerical methods for scalar conservation laws. Comput. Appl. Math. 14 (1995) 37-47.

[14] B. Cockburn and P.A. Gremaud, A priori error estimates for numerical methods for scalar conservation laws. Part I: The general approach. Math. Comput. 65 (1996) 533-573.

[15] B. Cockburn and G. Gripenberg, Continuous dependence on the nonlinearities of solutions of degenerate parabolic equations. J. Differential Equations 151 (1999) 231-251.

[16] W. Dörfler, Uniformly convergent finite-element methods for singularly perturbed convection-diffusion equations. Habilitationsschrift, Mathematische Fakultät, Freiburg (1998).

[17] K. Eriksson and C. Johnson, Adaptive streamline diffusion finite element methods for stationary convection-diffusion problems. Math. Comput. 60 (1993) 167-188.

[18] K. Eriksson and C. Johnson, Adaptive finite element methods for parabolic problems. II: Optimal error estimates in $L_{\infty} L_{2}$ and $L_{\infty} L_{\infty}$. SIAM J. Numer. Anal. 32 (1995) 706-740.

[19] K. Eriksson and C. Johnson, Adaptive finite element methods for parabolic problems. IV: Nonlinear Problems. SIAM J. Numer. Anal. 32 (1995) 1729-1749.

[20] S. Evje, K.H. Karlsen and N.H. Risebro, A continuous dependence result for nonlinear degenerate parabolic equations with spatial dependent flux function. Preprint, Department of Mathematics, Bergen University (2000).

[21] R. Eymard, T. Gallouët, M. Ghilani and R. Herbin, Error estimates for the approximate solution of a nonlinear hyperbolic equation given by finite volume schemes. IMA J. Numer. Anal. 18 (1998) 563-594.

[22] R. Eymard, T. Gallouët, R. Herbin and A. Michel, Convergence of a finite volume scheme for nonlinear degenerate parabolic equations. Preprint LATP 00-20, CMI, Provence University, Marseille (2000).

[23] P. Frolkovic, Maximum principle and local mass balance for numerical solutions of transport equations coupled with variable density flow. Acta Math. Univ. Comenian. 67 (1998) 137-157.

[24] J. Fuhrmann and H. Langmach, Stability and existence of solutions of time-implicit finite volume schemes for viscous nonlinear conservation laws. Preprint 437, Weierstraß-Institut, Berlin (1998).

[25] R. Helmig, Multiphase flow and transport processes in the subsurface: A contribution to the modeling of hydrosystems. Springer, Berlin, Heidelberg (1997).

[26] R. Herbin, An error estimate for a finite volume scheme for a diffusion-convection problem on a triangular mesh. Numer. Methods Partial Differential Equation 11 (1995) 165-173.

[27] P. Houston and E. Süli, Adaptive lagrange-galerkin methods for unsteady convection-dominated diffusion problems. Report 95/24, Numerical Analysis Group, Oxford University Computing Laboratory (1995).

[28] J. Jaffre, Décentrage et élements finis mixtes pour les équations de diffusion-convection. Calcolo 21 (1984) 171-197.

[29] V. John, J.M. Maubach and L. Tobiska, Nonconforming streamline-diffusion-finite-element-methods for convection-diffusion problems. Numer. Math. 78 (1997) 165-188. 
[30] C. Johnson, Finite element methods for convection-diffusion problems, in Proc. 5th Int. Symp. (Versailles, 1981), Computing methods in applied sciences and engineering $\mathbf{V}$ (1982) 311-323.

[31] K.H. Karlsen and N.H. Risebro, On the uniqueness and stability of entropy solutions of nonlinear degenerate parabolic equations with rough coefficients. Preprint 143, Department of Mathematics, Bergen University (2000).

[32] D. Kröner, Numerical schemes for conservation laws. Teubner, Stuttgart (1997).

[33] D. Kröner and M. Ohlberger, A posteriori error estimates for upwind finite volume schemes for nonlinear conservation laws in multi dimensions. Math. Comput. 69 (2000) 25-39.

[34] D. Kröner and M. Rokyta, A priori error estimates for upwind finite volume schemes in several space dimensions. Preprint 37 , Math. Fakultät, Freiburg (1996).

[35] S.N. Kruzkov, First order quasilinear equations in several independent variables. Math. USSR Sbornik 10 (1970) $217-243$.

[36] N.N. Kuznetsov, Accuracy of some approximate methods for computing the weak solutions of a first-order quasi-linear equation. USSR, Comput. Math. Math. Phys. 16 (1976) 159-193.

[37] J. Málek, J. Nečas, M. Rokyta and M. Růžička, Weak and measure-valued solutions to evolutionary PDEs, in Applied Mathematics and Mathematical Computation 13, Chapman and Hall, London, Weinheim, New York, Tokyo, Melbourne, Madras (1968).

[38] M. Marion and A. Mollard, An adaptive multi-level method for convection diffusion problems. ESAIM: M2AN 34 (2000) $439-458$.

[39] R.H. Nochetto, A. Schmidt and C. Verdi, A posteriori error estimation and adaptivity for degenerate parabolic problems. Math. Comput. 69 (2000) 1-24.

[40] M. Ohlberger, Convergence of a mixed finite element-finite volume method for the two phase flow in porous media. East-West J. Numer. Math. 5 (1997) 183-210.

[41] M. Ohlberger, A posteriori error estimates for finite volume approximations to singularly perturbed nonlinear convectiondiffusion equations. Numer. Math. 87 (2001) 737-761.

[42] Ch. Rohde, Entropy solutions for weakly coupled hyperbolic systems in several space dimensions. Z. Angew. Math. Phys. 49 (1998) 470-499.

[43] Ch. Rohde, Upwind finite volume schemes for weakly coupled hyperbolic systems of conservation laws in 2D. Numer. Math. 81 (1998) 85-123.

[44] H.-G. Roos, M. Stynes and L. Tobiska, Numerical methods for singularly perturbed differential equations. Convection-diffusion and flow problems, in Springer Ser. Comput. Math. 24, Springer-Verlag, Berlin (1996).

[45] E. Tadmor, Local error estimates for discontinuous solutions of nonlinear hyperbolic equations. SIAM J. Numer. Anal. 28 (1991) 891-906.

[46] R. Verfürth, A review of a posteriori error estimation and adaptive mesh-refinement techniques, in Wiley-Teubner Ser. Adv. Numer. Math., Teubner, Stuttgart (1996).

[47] R. Verfürth, A posteriori error estimators for convection-diffusion equations. Numer. Math. 80 (1998) 641-663.

[48] J.P. Vila, Convergence and error estimates in finite volume schemes for general multi-dimensional scalar conservation laws. I Explicit monotone schemes. ESAIM: M2AN 28 (1994) 267-295.

To access this journal online:

www.edpsciences.org 